\title{
Toeplitz and slant Toeplitz operators on the polydisk
}

\author{
Munmun Hazarika and Sougata Marik \\ Department of Mathematical Sciences, Tezpur University, Tezpur, India
}

\begin{abstract}
For $n \geq 1$, let $\mathbb{D}^{n}$ be the polydisk in $\mathbb{C}^{n}$, and let $\mathbb{T}^{n}$ be the $n$-torus. $L^{2}\left(\mathbb{T}^{n}\right)$ denotes the space of Lebesgue square integrable functions on $\mathbb{T}^{n}$. In this paper we define slant Toeplitz operators on $L^{2}\left(\mathbb{T}^{n}\right)$. Besides giving a necessary and sufficient condition for an operator on $L^{2}\left(\mathbb{T}^{n}\right)$ to be slant Toeplitz, we also establish several properties of slant Toeplitz operators.
\end{abstract}

Keywords Slant Toeplitz operator, Laurent matrix of level n, Toeplitz matrix of level n, Hyponormal operator Paper type Original Article

\section{Introduction}

Let $\mathbb{D}$ denote the open unit disk and $\mathbb{T}$ denote the unit circle in the complex plane $\mathbb{C}$. For $n \geq 1$, let $\mathbb{D}^{n}$ be the polydisk in $\mathbb{C}^{n}$, and let $\mathbb{T}^{n}$ be the $n$-torus which is the distinguished boundary of $\mathbb{D}^{n}$. Also let $d \sigma$ be the normalized Haar measure (or Lebesgue measure) on $\mathbb{T}^{n}$. In the sequel, $z$ will always denote a vector $z=\left(z_{1}, \ldots, z_{n}\right)$ in $\mathbb{C}^{n}$, and for $m=\left(m_{1}, \ldots m_{n}\right) \in \mathbb{Z}^{n}, z^{m}:=z_{1}^{m_{1}} \ldots z_{n}^{m_{n}}$ and $|m|:=m_{1}+\ldots+m_{n}$. Also for $\lambda \in \mathbb{Z}$ we denote the $n$-tuple $(\lambda, \ldots, \lambda)$ as $\lambda$ and $z^{\lambda}:=z_{1}^{\lambda} \ldots z_{n}^{\lambda}$. In particular, $z=z_{1} \ldots z_{n}$.

$L^{2}\left(\mathbb{T}^{n}\right)$ is the space of Lebesgue square integrable functions on $\mathbb{T}^{n}$ with respect to $d \sigma$. Thus,

$$
L^{2}\left(\mathbb{T}^{n}\right)=\left\{f:\left.\mathbb{T}^{n} \mapsto \mathbb{C}\left|f(z)=\sum_{m \in \mathbb{Z}^{n}} f_{m} z^{m}, \sum_{m \in \mathbb{Z}^{n}}\right| f_{m}\right|^{2}<\infty\right\} \text { and for } f, g \in
$$
$L^{2}\left(\mathbb{T}^{n}\right),\langle f, g\rangle:=\int_{\mathbb{T}^{n}} f(z) \overline{g(z)} d \sigma(z) \cdot L^{\infty}\left(\mathbb{T}^{n}\right)$ is the subspace of $L^{2}\left(\mathbb{T}^{n}\right)$, consisting of all $\varphi \in L^{2}\left(\mathbb{T}^{n}\right)$ having the following two properties:

1. $\varphi f \in L^{2}\left(\mathbb{T}^{n}\right) \forall f \in L^{2}\left(\mathbb{T}^{n}\right)$.

2. $\exists c>0$ such that $\|\varphi f\| \leq c\|f\| \forall f \in L^{2}\left(\mathbb{T}^{n}\right)$.

For $\varphi \in L^{\infty}\left(\mathbb{T}^{n}\right),\|\varphi\|_{\infty}$ is defined as $\|\varphi\|_{\infty}=\inf \left\{c>0 \mid\|\varphi f\| \leq c\|f\| \forall f \in L^{2}\left(\mathbb{T}^{n}\right)\right\}$.

\section{JEL Classification - primary 47B37, secondary 47B20}

(C) Munmun Hazarika and Sougata Marik. Published in the Arab Journal of Mathematical Sciences. Published by Emerald Publishing Limited. This article is published under the Creative Commons Attribution (CCBY 4.0) license. Anyone may reproduce, distribute, translate and create derivative works of this article (for both commercial and non-commercial purposes), subject to full attribution to the original publication and authors. The full terms of this license may be seen at http://creativecommons. org/licences/by/4.0/legalcode

We thank the unknown referee for his/her valuable suggestions which helped us improve the paper.

The publisher wishes to inform readers that the article "Toeplitz and slant Toeplitz operators on the polydisk" was originally published by the previous publisher of the Arab Journal of Mathematical Sciences and the pagination of this article has been subsequently changed. There has been no change to the content of the article. This change was necessary for the journal to transition from the previous publisher to the new one. The publisher sincerely apologises for any inconvenience caused. To access and cite this article, please use Hazarika, M., Marik, S. (2019), "Toeplitz and slant Toeplitz operators on the polydisk", Arab Journal of Mathematical Sciences, Vol. 27 No. 1, pp. 73-93. The original publication date for this paper was 25/02/2019.
Received 12 March 2018 Revised 13 February 2019 Accepted 18 February 2019 
AJMS

27,1

The Hardy space $H^{2}\left(\mathbb{D}^{n}\right)$ consists of all holomorphic functions $f$ on $\mathbb{D}^{n}$ such that $\sup _{0<r<1} \int_{\mathbb{T}^{n}}|f(r z)|^{2} d \sigma(z)<\infty$. For every function $f \in H^{2}\left(\mathbb{D}^{n}\right)$, the radial limit $\lim _{r \rightarrow 1^{-}} f(r z)$ exists for almost every $z \in \mathbb{T}^{n}$ [20]. If we denote this radial limit by $f(z)$, then the Hardy space $H^{2}\left(\mathbb{D}^{n}\right)$ can be isometrically identified with the closure of the polynomials in $L^{2}\left(\mathbb{T}^{n}\right)$. Thus we can now define $P$ to be the orthogonal projection of $L^{2}\left(\mathbb{T}^{n}\right)$ onto $H^{2}\left(\mathbb{D}^{n}\right)$.

M. C. Ho [11] defined slant Toeplitz operators on $L^{2}(\mathbb{T})$ as those operators whose matrix representation with respect to an orthonormal basis can be obtained by eliminating every other row of a doubly infinite Toeplitz matrix. These types of operators appear frequently in wavelet analysis as their spectral properties have a connection with the smoothness of wavelets. The study of slant Toeplitz operators paved the way to the introduction of more new classes of operators over various function spaces, like $k$ th order slant Toeplitz operators, essentially slant Toeplitz operators, weighted slant Toeplitz operators and so on. For relevant results on slant Toeplitz operators and their generalizations, we refer the reader to $[1,3,5,10-13,17,18,23]$.

In this paper we define slant Toeplitz operators on $L^{2}\left(\mathbb{T}^{n}\right)$, for $n \geq 1$. Motivated by the matricial definition of slant Toeplitz operators on $L^{2}(\mathbb{T})$, as given by M. C. Ho [11], we define a slant Toeplitz matrix of level-n, and subsequently show that an operator on $L^{2}\left(\mathbb{T}^{n}\right)$ is a slant Toeplitz operator if and only if it can be represented as a slant Toeplitz matrix of level- $n$. For this we first define a Laurent matrix of level- $n$ and establish its relation to a Laurent operator on $L^{2}\left(\mathbb{T}^{n}\right)$.

\section{Laurent matrix of level $\boldsymbol{n}$}

Definition 2.1. For $\varphi \in L^{\infty}\left(\mathbb{T}^{n}\right)$, the Laurent operator $M_{\varphi}$ is defined as the multiplication by $\varphi$. That is, $M_{\varphi} f=\varphi$. $f \forall f \in L^{2}\left(\mathbb{T}^{n}\right)$.

Remark 2.2 (i). For $\varphi \in L^{\infty}\left(\mathbb{T}^{n}\right)$, we can easily show that $M_{\varphi}^{*}=M_{\bar{\varphi}}$.

(ii) Let $i$ be an integer such that $1 \leq i \leq n$. If $\varphi(z)=z_{i}$ then $M_{\varphi} f=z_{i} f \forall f \in L^{2}\left(\mathbb{T}^{n}\right)$. In this case we denote $M_{\varphi}$ as $M_{z_{i}}$.

Definition 2.3 (Laurent Matrix of Level n). Consider scalars $\left\{a_{k}\right\}_{k \in \mathbb{Z}^{n}}$. A matrix of the type

$$
\left(\begin{array}{ccccc}
\ddots & \vdots & \vdots & \vdots & \vdots \\
\ldots & a_{\left(0, k_{2}, \ldots, k_{n}\right)} & a_{\left(-1, k_{2}, \ldots, k_{n}\right)} & a_{\left(-2, k_{2}, \ldots, k_{n}\right)} & \ldots \\
\ldots & a_{\left(1, k_{2}, \ldots, k_{n}\right)} & {\left[a_{\left(0, k_{2}, \ldots, k_{n}\right)}\right]} & a_{\left(-1, k_{2}, \ldots, k_{n}\right)} & \ldots \\
\ldots & a_{\left(2, k_{2}, \ldots, k_{n}\right)} & a_{\left(1, k_{2}, \ldots, k_{n}\right)} & a_{\left(0, k_{2}, \ldots, k_{n}\right)} & \ldots \\
\vdots & \vdots & \vdots & \vdots & \ddots
\end{array}\right)
$$

is said to be Laurent matrix of level 1 and is denoted as $L_{k_{2}, \ldots, k_{n}}^{(1)}$.

A block matrix of the type

$$
\left(\begin{array}{ccccc}
\ddots & \vdots & \vdots & \vdots & \vdots \\
\ldots & L_{0, k_{3}, \ldots, k_{n}}^{(1)} & L_{-1, k_{3}, \ldots, k_{n}}^{(1)} & L_{-2, k_{3}, \ldots, k_{n}}^{(1)} & \ldots \\
\ldots & L_{1, k_{3}, \ldots, k_{n}}^{(1)} & {\left[L_{0, k_{3}, \ldots, k_{n}}^{(1)}\right.} & L_{-1, k_{3}, \ldots, k_{n}}^{(1)} & \ldots \\
\ldots & L_{2, k_{3}, \ldots, k_{n}}^{(1)} & L_{1, k_{3}, \ldots, k_{n}}^{(1)} & L_{0, k_{3}, \ldots, k_{n}}^{(1)} & \ldots \\
\vdots & \vdots & \vdots & \vdots & \ddots
\end{array}\right)
$$

is said to be Laurent matrix of level 2 and is denoted by $L_{k_{3}, \ldots, k_{n}}^{(2)}$. 
A block matrix of the type

$$
\left(\begin{array}{ccccc}
\ddots & \vdots & \vdots & \vdots & \vdots \\
\ldots & L_{0, k_{4}, \ldots, k_{n}}^{(2)} & L_{-1, k_{4}, \ldots, k_{n}}^{(2)} & L_{-2, k_{4}, \ldots, k_{n}}^{(2)} & \ldots \\
\ldots & L_{1, k_{4}, \ldots, k_{n}}^{(2)} & {\left[L_{0, k_{4}, \ldots, k_{n}}^{(2)}\right]} & L_{-1, k_{4}, \ldots, k_{n}}^{(2)} & \ldots \\
\ldots & L_{2, k_{4}, \ldots, k_{n}}^{(2)} & L_{1, k_{4}, \ldots, k_{n}}^{(2)} & L_{0, k_{4}, \ldots, k_{n}}^{(2)} & \ldots \\
\vdots & \vdots & \vdots & \vdots & \ddots
\end{array}\right)
$$

is said to be Laurent matrix of level 3 and is denoted as $L_{k_{4}, \ldots, k_{n}}^{(3)}$. Continuing, we get a block matrix of type

$$
\left(\begin{array}{ccccc}
\ddots & \vdots & \vdots & \vdots & \vdots \\
\ldots & L_{0}^{(n-1)} & L_{-1}^{(n-1)} & L_{-2}^{(n-1)} & \ldots \\
\ldots & L_{1}^{(n-1)} & {\left[L_{0}^{(n-1)}\right]} & L_{-1}^{(n-1)} & \ldots \\
\ldots & L_{2}^{(n-1)} & L_{1}^{(n-1)} & L_{0}^{(n-1)} & \ldots \\
\vdots & \vdots & \vdots & \vdots & \ddots
\end{array}\right)
$$

called Laurent matrix of level $n$ denoted as $L^{(n)}$.

For example when $n=4$ we consider the sequence $\left\{a_{k}\right\}_{k \in \mathbb{Z}^{4}}$ given by

$$
a\left(k_{1}, k_{2}, k_{3}, k_{4}\right)= \begin{cases}1, & \text { if } k_{1} \text { is even; } \\ 2, & \text { if } k_{1} \text { is odd, } k_{2} \text { is even; } \\ 3, & \text { if } k_{1}, k_{2} \text { are odd, } k_{3} \text { is even; } \\ 4, & \text { if } k_{1}, k_{2}, k_{3} \text { are odd, } k_{4} \text { is even; } \\ 5, & \text { if } k_{1}, k_{2}, k_{3}, k_{4} \text { are odd. }\end{cases}
$$

Then

$$
L_{k_{2}, k_{3}, k_{4}}^{(1)}=\left(\begin{array}{ccccc}
\ddots & \vdots & \vdots & \vdots & \vdots \\
\cdots & 1 & j & 1 & \ldots \\
\cdots & j & {[1]} & j & \ldots \\
\cdots & 1 & j & 1 & \ldots \\
\vdots & \vdots & \vdots & \vdots & \ddots
\end{array}\right)
$$

where $j= \begin{cases}2, & \text { if } k_{2} \text { even; } \\ 3, & \text { if } k_{2} \text { odd, } k_{3} \text { even; } \\ 4, & \text { if } k_{2}, k_{3} \text { odd, } k_{4} \text { even; } \\ 5, & \text { if } k_{2}, k_{3}, k_{4} \text { odd. }\end{cases}$

If we denote $L_{k_{2}, k_{3}, k_{4}}^{(1)}$ as $A, B, C, D$ for $j$ taking values $2,3,4,5$ respectively, then

$$
L_{k_{3}, k_{4}}^{(2)}=\left(\begin{array}{ccccc}
\ddots & \vdots & \vdots & \vdots & \vdots \\
\ldots & A & B & A & \ldots \\
\ldots & B & {[A]} & B & \ldots \\
\ldots & A & B & A & \ldots \\
\vdots & \vdots & \vdots & \vdots & \ddots
\end{array}\right)
$$


$\begin{aligned} & \text { AJMS } \\ & 27,1\end{aligned} L_{k_{3}, k_{4}}^{(2)}=\left(\begin{array}{ccccc}\ddots & \vdots & \vdots & \vdots & \vdots \\ \ldots & A & C & A & \ldots \\ \ldots & C & {[A]} & C & \ldots \\ \ldots & A & C & A & \ldots \\ \vdots & \vdots & \vdots & \vdots & \ddots\end{array}\right)$

if $k_{3}$ is odd but $k_{4}$ is even; and

$$
L_{k_{3}, k_{4}}^{(2)}=\left(\begin{array}{ccccc}
\ddots & \vdots & \vdots & \vdots & \vdots \\
\cdots & A & D & A & \cdots \\
\cdots & D & {[A]} & D & \cdots \\
\cdots & A & D & A & \cdots \\
\vdots & \vdots & \vdots & \vdots & \ddots
\end{array}\right)
$$

if $k_{3}$ and $k_{4}$ are both odd. Let us denote these three matrices by $E, F, G$ respectively. Then,

$$
L_{k_{4}}^{(3)}=\left(\begin{array}{ccccc}
\ddots & \vdots & \vdots & \vdots & \vdots \\
\cdots & E & F & E & \cdots \\
\cdots & F & {[E]} & F & \cdots \\
\cdots & E & F & E & \cdots \\
\vdots & \vdots & \vdots & \vdots & \ddots
\end{array}\right)
$$

if $k_{4}$ is even; and

$$
L_{k_{4}}^{(3)}=\left(\begin{array}{ccccc}
\ddots & \vdots & \vdots & \vdots & \vdots \\
\cdots & E & G & E & \cdots \\
\cdots & G & {[E]} & G & \cdots \\
\cdots & E & G & E & \cdots \\
\vdots & \vdots & \vdots & \vdots & \ddots
\end{array}\right)
$$

if $k_{4}$ is odd. If we denote these two matrices by I and J respectively, then

$$
L^{(4)}=\left(\begin{array}{ccccc}
\ddots & \vdots & \vdots & \vdots & \vdots \\
\cdots & I & J & I & \cdots \\
\cdots & J & {[I]} & J & \cdots \\
\cdots & I & J & I & \cdots \\
\vdots & \vdots & \vdots & \vdots & \ddots
\end{array}\right)
$$

Notation to be followed:

1. $\mathbb{Z}, \mathbb{Z}_{+}$and $\mathbb{N}$ denote the set of integers, set of non negative integers, and set of positive integers respectively.

2. For $k \in \mathbb{Z}^{n}$, let $e_{k}(z):=z^{k}$. Then $Q=\left\{e_{k}\right\}_{k \in Z^{n}}$ is an orthonormal basis for $L^{2}\left(\mathbb{T}^{n}\right)$, and $\left\{e_{k}\right\}_{k \in \mathbb{Z}_{+}^{n}}$ is an orthonormal basis for $H^{2}\left(\mathbb{D}^{n}\right)$, as shown in [20].

3. For $k=\left(k_{1}, \ldots, k_{n}\right) \in \mathbb{Z}^{n}$, we say that $k$ is even if each $k_{i}$ is even. Otherwise $k$ is said to be odd. 
4. $S:=\left\{\left(t_{1}, \ldots, t_{n}\right) \in \mathbb{Z}^{n}\right.$ : each $t_{i}$ is either 0 or 1$\}$. If $o(S)$ denotes the order of the set $S$, then $o(S)=2^{n}$.

5. For $j \in[1, n] \cap \mathbb{Z}$, let $\epsilon_{j}:=\left(x_{1}, \ldots, x_{n}\right)$ where $x_{i}=\delta_{i j}$.

6. For $j \in(1, n] \cap \mathbb{Z}$, and arbitrarily fixed $k_{j}, k_{j+1}, \ldots, k_{n} \in \mathbb{Z}$, let $Q_{\left[k_{j}, \ldots, k_{n}\right]}=\left\{e_{\left(k_{1}, \ldots, k_{n}\right)}\right.$ : $k_{i} \in \mathbb{Z}$ for $\left.1 \leq i<j\right\}$. Then $Q_{\left[k_{j}, \ldots, k_{n}\right]}$ is an orthonormal basis for $L^{2}\left(\mathbb{T}^{j-1}\right)$.

7. For $1<j \leq n$, and $k_{i} \in \mathbb{Z}$ with $j \leq i \leq n$, we often use the notation $\mathcal{H}_{j-1,\left(k_{j}, \ldots, k_{n}\right)}$ for the space $L^{2}\left(\mathbb{T}^{j-1}\right)$ to convey that the basis being considered is $Q_{\left[k_{j}, \ldots, k_{n}\right]}$.

Theorem 2.4. For $i=1,2, \ldots, n, M_{z_{i}}^{*}=M_{\bar{z}_{i}}$ and $M_{z_{i}}^{*} e_{k}=e_{k-\epsilon_{i}} \forall k \in \mathbb{Z}^{n}$.

Proof. Let $f \in L^{2}\left(\mathbb{T}^{n}\right)$ and $f(z)=\sum_{t \in \mathbb{Z}^{n}} a_{t} z^{t}$. Then for $i=1, \ldots, n$ and $k \in \mathbb{Z}^{n}$, we have,

$$
\left\langle M_{z_{i}} f, e_{k}\right\rangle=\left\langle z_{i} f(z), z^{k}\right\rangle=\sum_{t \in \mathbb{Z}^{n}} a_{t}\left\langle z^{t+\epsilon_{i}}, z^{k}\right\rangle=a_{k-\epsilon_{i}}=\left\langle f, e_{k-\epsilon_{i}}\right\rangle .
$$

Thus, $M_{z_{i}}^{*} e_{k}=e_{k-\epsilon_{i}}=\bar{z}_{i} e_{k}$ which implies that $M_{z_{i}}^{*} f(z)=\bar{z}_{i} f(z)$, so that $M_{z_{i}}^{*}=M_{\bar{z}_{i}}$.

Remark 2.5. (i) $M_{z_{i}} e_{k}=e_{k+\epsilon_{i}}$, for $1 \leq i \leq n$ and $k \in \mathbb{Z}^{n}$. (ii) $M_{z_{i}} M_{z_{i}}^{*}=I=M_{z_{i}}^{*} M_{z_{i}} \forall 1 \leq i \leq n$.

Theorem 2.6. A bounded linear operator $A$ on $L^{2}\left(\mathbb{T}^{n}\right)$ can be represented as a Laurent matrix of level-n if and only if $\left\langle A e_{k+\epsilon_{j}}, e_{t+\epsilon_{j}}\right\rangle=\left\langle A e_{k}, e_{t}\right\rangle \forall k, t \in \mathbb{Z}^{n}, 1 \leq j \leq n$.

Proof. Let us assume first that $\left\langle A e_{k+\epsilon_{j}}, e_{t+\epsilon_{j}}\right\rangle=\left\langle A e_{k}, e_{t}\right\rangle \forall k, t \in \mathbb{Z}^{n}, 1 \leq j \leq n$. Also let $\left\{a_{\eta, \xi}\right\}_{\eta, \xi \in \mathbb{Z}^{n}}$ be scalars such that

$$
A e_{\xi}=\sum_{\eta \in \mathbb{Z}^{n}} a_{\eta, \xi} e_{\eta} \forall \xi \in \mathbb{Z}^{n}
$$

Step I: Let $k=\left(k_{1}, \ldots, k_{n}\right) \in \mathbb{Z}^{n}$ and $t=\left(t_{1}, \ldots, t_{n}\right) \in \mathbb{Z}^{n}$ be arbitrarily chosen. Keeping $\left(k_{2}, \ldots, k_{n}\right)$ and $\left(t_{2}, \ldots, t_{n}\right)$ fixed, if we vary $k_{1}$ and $t_{1}$, then $\left\langle A e_{k+\epsilon_{1}}, e_{t+\epsilon_{1}}\right\rangle=\left\langle A e_{k}, e_{t}\right\rangle$ implies that $a_{t+\epsilon_{1}, k+\epsilon_{1}}=a_{t, k} \forall k_{1}, t_{1} \in \mathbb{Z}$. This means that $A: \mathcal{H}_{1,\left[k_{2}, \ldots, k_{n}\right]} \mapsto \mathcal{H}_{1,\left[t_{2}, \ldots, t_{n}\right]}$ can be represented as a Laurent matrix of level-1, which we denote as $M_{\left(t_{2}, \ldots, t_{n}\right)\left(k_{2}, \ldots, k_{n}\right)}^{1}$.

Step II: Here we keep $\left(k_{3}, \ldots, k_{n}\right)$ and $\left(t_{3}, \ldots, t_{n}\right)$ fixed and vary $k_{2}$ and $t_{2}$. Then $\left\langle A e_{k+\epsilon_{2}}, e_{t+\epsilon_{2}}\right\rangle=\left\langle A e_{k}, e_{t}\right\rangle$ implies that $M_{\left(t_{2}+1, t_{3}, \ldots, t_{n}\right)\left(k_{2}+1, k_{3}, \ldots, k_{n}\right)}^{1}=M_{\left(t_{2}, \ldots, t_{n}\right)\left(k_{2}, \ldots, k_{n}\right)}^{1}$, and so $A$ :

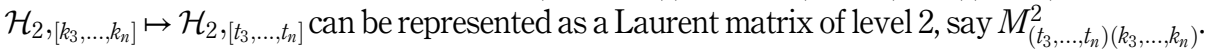

Step III: Keeping $\left(k_{4}, \ldots, k_{n}\right)$ and $\left(t_{4}, \ldots, t_{n}\right)$ fixed, and using the fact that $\left\langle A e_{k+\epsilon_{3}}, e_{t+\epsilon_{3}}\right\rangle=\left\langle A e_{k}, e_{t}\right\rangle$, we get $M_{\left(t_{3}+1, t_{4}, \ldots, t_{n}\right)\left(k_{3}+1, k_{4}, \ldots, k_{n}\right)}^{2}=M_{\left(t_{3}, \ldots, t_{n}\right)\left(k_{3}, \ldots, k_{n}\right)}^{2}$, and so $A$ : $\mathcal{H}_{3},\left[k_{4}, \ldots, k_{n}\right] \mapsto \mathcal{H}_{3},\left[t_{4}, \ldots, t_{n}\right]$ can be represented as a Laurent matrix of level 3 . Continuing in the same way, after $n$ steps we finally arrive at the fact that $A: L^{2}\left(\mathbb{T}^{n}\right) \mapsto L^{2}\left(\mathbb{T}^{n}\right)$ can be represented as a Laurent matrix of level- $n$.

To prove the converse we assume that $A$ can be represented as a Toeplitz matrix of level-n. Hence for $k=\left(k_{1}, \ldots, k_{n}\right)$ and $t=\left(t_{1}, \ldots, t_{n}\right)$ arbitrarily chosen, $A: \mathcal{H}_{j-1,\left[k_{j}, \ldots, k_{n}\right]}$ $\mapsto \mathcal{H}_{j-1\left[t_{j}, \ldots, t_{n}\right]}$ can be represented as a Toeplitz matrix of level $(j-1)$, denoted as $M_{\left(t_{j} \ldots, t_{n}\right)\left(k_{j} \ldots, k_{n}\right)}^{j-1}$, for each $j=2, \ldots, n$. This in turn implies that $\left\langle A e_{k+\epsilon_{j}}, e_{t+\epsilon_{j}}\right\rangle=\left\langle A e_{k}, e_{t}\right\rangle$ for $j=1, \ldots, n-1$.

Finally to show that $\left\langle A e_{k+\epsilon_{n}}, e_{t+\epsilon_{n}}\right\rangle=\left\langle A e_{k}, e_{t}\right\rangle$ we consider the $(n-1)$-torus $L^{2}\left(\mathbb{T}^{n-1}\right)$. For each $k_{n} \in \mathbb{Z}, L^{2}\left(\mathbb{T}^{n-1}\right)$ is an isomorphic copy of $\mathcal{H}_{n-1,\left(k_{n}\right)}$, and hence the $n$-torus $L^{2}\left(\mathbb{T}^{n}\right)$ can be
Toeplitz and slant Toeplitz operators 
AJMS

27,1

decomposed as $L^{2}\left(\mathbb{T}^{n}\right)=\bigoplus_{k_{n} \in \mathbb{Z}} \mathcal{H}_{n-1},\left[k_{n}\right]$. Therefore, $A: L^{2}\left(\mathbb{T}^{n}\right) \mapsto L^{2}\left(\mathbb{T}^{n}\right)$ can be expressed as a matrix where the $\left(t_{n}, k_{n}\right)^{t h}$ entry is $M_{t_{n}, k_{n}}^{n-1}$, and we also have $M_{t_{n}+1, k_{n}+1}^{n-1}=M_{t_{n}, k_{n}}^{n-1}$. This finally implies that $\left\langle A e_{k+\epsilon_{n}}, e_{t+\epsilon_{n}}\right\rangle=\left\langle A e_{k}, e_{t}\right\rangle$.

Theorem 2.7. Let $A$ be a bounded linear operator on $L^{2}\left(\mathbb{T}^{n}\right)$. Then the following conditions are equivalent:

1. A can be represented as a Laurent matrix of level-n

2. A commutes with $M_{z_{j}}$ for $1 \leq j \leq n$

3. $M_{z^{k}} A=A M_{z^{k}} \forall k \in \mathbb{Z}^{n}$

Proof. $(1) \Rightarrow(2)$

Since $A$ can be represented as a Laurent matrix of level- $n$, so by Theorem 2.6 we have, $\left\langle A e_{k+\epsilon_{i}}, e_{t}\right\rangle=\left\langle A e_{k}, e_{t-\epsilon_{j}}\right\rangle \forall k, t \in \mathbb{Z}^{n}, 1 \leq j \leq n$. Applying Remark 2.5(i) and Theorem 2.4 to this relation we further get $\left\langle A M_{z_{j}} e_{k}, e_{t}\right\rangle=\left\langle M_{z_{j}} A e_{k}, e_{t}\right\rangle \forall k, t \in \mathbb{Z}^{n}, 1 \leq j \leq n$. Thus, $A M_{z_{j}}=M_{z_{j}} A \forall 1 \leq j \leq n$.

$(2) \Rightarrow(3)$

Suppose $M_{z_{i}} A=A M_{z_{i}} \forall 1 \leq i \leq n$. Then for $k=\left(k_{1}, \ldots, k_{n}\right) \in \mathbb{Z}^{n}$, we have $M_{z^{k}} A=$ $M_{z_{1}}^{k_{1}} \ldots M_{z_{n}}^{k_{n}} A=A M_{z_{1}}^{k_{1}} \ldots M_{z_{n}}^{k_{n}}=\bar{A} M_{z^{k}}$.

$(3) \Rightarrow(1)$

As $M_{z^{k}} A=A M_{z^{k}} \forall k \in \mathbb{Z}^{n}$, so taking $k=\epsilon_{i}$ we get $M_{z_{i}} A=A M_{z_{i}}$ for each $i=1, \ldots, n$. Now for $k, t \in \mathbb{Z}^{n}$, we have $\left\langle A e_{k+\epsilon_{j}}, e_{t+\epsilon_{j}}\right\rangle=\left\langle A M_{z_{j}} e_{k}, M_{z_{l}} e_{t}\right\rangle=\left\langle M_{z_{j}} A e_{k}, M_{z_{j}} e_{t}\right\rangle$, and using Remark 2.5(ii) we get $\left\langle A e_{k+\epsilon_{j}}, e_{t+\epsilon_{j}}\right\rangle=\left\langle A e_{k}, e_{t}\right\rangle$ for all $k, t \in \mathbb{Z}^{n}$. Hence applying Theorem 2.6 we conclude that $A$ can be represented as a Laurent matrix of level-n.

Theorem 2.8. A bounded linear operator on $L^{2}\left(\mathbb{T}^{n}\right)$ is a Laurent operator if and only if it can be represented as a Laurent matrix of level $n$.

Proof. For $\varphi \in L^{\infty}\left(\mathbb{T}^{n}\right), M_{\varphi} M_{z_{i}}=M_{z_{i}} M_{\varphi} \forall 1 \leq i \leq n$, and so by Theorem 2.7, $M_{\varphi}$ can be represented as a Laurent matrix of level $n$.

Conversely, let $\varphi=A e_{0}$, so that $\varphi \in L^{2}\left(\mathbb{T}^{n}\right)$. We first show that $\varphi$ is bounded.

We have $\|\varphi\|=\left\|A e_{0}\right\| \leq\|A\|\left\|e_{0}\right\|$. Again, $\left\|\varphi^{2}\right\|=\left\|A e_{0} . \varphi\right\| \leq\|A\|^{2}\left\|e_{0}\right\|$. Repeating this argument inductively, we get $\left\|\varphi^{m}\right\| \leq\|A\|^{m}\left\|e_{0}\right\|$ for every positive integer $m$. This norm

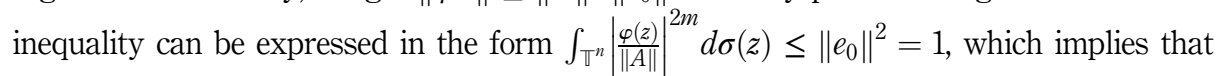
$\left|\frac{\varphi(z)}{\|A\|}\right| \leq 1$ almost everywhere. Thus $|\varphi(z)| \leq\|A\|$ almost everywhere which shows that $\varphi$ is bounded. Hence $\varphi \in L^{\infty}\left(\mathbb{T}^{n}\right)$ and $\|\varphi\|_{\infty} \leq\|A\|$.

Next we will show that $A=M_{\varphi}$. As $A$ can be represented as a Laurent matrix of level $n$, so by Theorem 2.7, $A M_{z^{k}}=M_{z^{k}} A \forall k \in \mathbb{Z}^{n}$. Therefore,

$$
\varphi(z) z^{k}=z^{k} \varphi(z)=M_{z^{k}} \varphi(z)=M_{z^{k}} A e_{0}(z)=A M_{z^{k}} e_{0}(z)=A z^{k} .
$$

Thus $A f=\varphi f$ for every polynomial $f$ in $z_{1}, \ldots, z_{n}$.

Let $f \in L^{2}\left(\mathbb{T}^{n}\right)$. Then there exist polynomials $f_{m}$ in $z_{1}, \ldots, z_{n}$ such that $f_{m} \rightarrow f$. This implies that $A f_{m} \rightarrow A f$ and $\varphi f_{m} \rightarrow \varphi f$, and since $A f_{m}=\varphi f_{m} \forall m$, so $A f=\varphi f$. Equivalently, $A=M_{\varphi}$.

The following corollary follows immediately from Theorems 2.6-2.8. 
Corollary 2.9. Let $A$ be a bounded linear operator on $L^{2}\left(\mathbb{T}^{n}\right)$. Then the following conditions are equivalent:

1. A is a Laurent operator

2. $\left\langle A e_{k+\epsilon_{j}}, e_{t+\epsilon_{j}}\right\rangle=\left\langle A e_{k}, e_{t}\right\rangle \forall k, t \in \mathbb{Z}^{n}, 1 \leq j \leq n$

3. $M_{z_{i}} A=A M_{z_{i}} \forall 1 \leq i \leq n$.

4. $M_{z^{k}} A=A M_{z^{k}} \forall k \in \mathbb{Z}^{n}$.

The proof being obvious is omitted.

\section{Toeplitz matrix of level $n$}

Definition 3.1. For $\varphi \in L^{\infty}\left(\mathbb{T}^{n}\right)$, the Toeplitz operator $T_{\varphi}$ is defined as the compression of $M_{\varphi}$ to $H^{2}\left(\mathbb{D}^{n}\right)$. That is, $T_{\varphi} f=P(\varphi . f) \forall f \in H^{2}\left(\mathbb{D}^{n}\right)$, where $P$ is the orthogonal projection of $L^{2}\left(\mathbb{T}^{n}\right)$ onto $H^{2}\left(\mathbb{D}^{n}\right)$. Thus,

$$
\left(T_{\varphi} f\right)(z)=P(\varphi(z) \cdot f(z)) \forall f \in H^{2}\left(\mathbb{D}^{n}\right), z \in \mathbb{T}^{n} .
$$

Toeplitz operators on $H^{2}\left(\mathbb{D}^{n}\right)$ have been earlier discussed by several authors. For the convenience of the reader we mention here a few significant references [4,6-8,14,16,19,21]. In the present work we give a matricial definition of Toeplitz operators on $H^{2}\left(\mathbb{D}^{n}\right)$. It may be mentioned that block Toeplitz operators with matrix symbol $F \in L^{\infty}\left(\mathbb{C}^{n \times n}\right)$ was first discussed in [9]. Again in [19] we come across block matrix representation of Toeplitz and Hankel operators on $L^{2}\left(\mathbb{T}^{n}\right)$. In [15] the authors have used lexicographic ordering on $\mathbb{Z}_{+}^{2}$ for matrix representation of Toeplitz operators on $L^{2}\left(\mathbb{T}^{n}\right)$. These references motivated us to propose our definition of a Toeplitz matrix of level- $n$. However, our approach is significantly different from what is done in earlier works. We first propose the definition of a Toeplitz matrix of level $n$ and then show that an operator on $H^{2}\left(\mathbb{D}^{n}\right)$ is a Toeplitz operator if and only if it can be represented as a Toeplitz matrix of level $n$.

Definition 3.2 (Toeplitz Matrix of Level $n$ ). Consider a sequence of scalars $\left\{a_{k}\right\}_{k \in \mathbb{Z}^{n}}$. A matrix of the type

$$
\left(\begin{array}{cccc}
a_{\left(0, k_{2}, \ldots, k_{n}\right)} & a_{\left(-1, k_{2}, \ldots, k_{n}\right)} & a_{\left(-2, k_{2}, \ldots, k_{n}\right)} & \ldots \\
a_{\left(1, k_{2}, \ldots, k_{n}\right)} & a_{\left(0, k_{2}, \ldots, k_{n}\right)} & a_{\left(-1, k_{2}, \ldots, k_{n}\right)} & \ldots \\
a_{\left(2, k_{2}, \ldots, k_{n}\right)} & a_{\left(1, k_{2}, \ldots, k_{n}\right)} & a_{\left(0, k_{2}, \ldots, k_{n}\right)} & \ldots \\
\vdots & \vdots & \vdots & \ddots
\end{array}\right)
$$

is said to be a Toeplitz matrix of level 1 , denoted as $T_{k_{2}, \ldots, k_{n}}^{(1)}$.

A block matrix of the type

$$
\left(\begin{array}{cccc}
T_{0, k_{3}, \ldots, k_{n}}^{(1)} & T_{-1, k_{3}, \ldots, k_{n}}^{(1)} & T_{-2, k_{3}, \ldots, k_{n}}^{(1)} & \cdots \\
T_{1, k_{3}, \ldots, k_{n}}^{(1)} & T_{0, k_{3}, \ldots, k_{n}}^{(1)} & T_{-1, k_{3}, \ldots, k_{n}}^{(1)} & \cdots \\
T_{2, k_{3}, \ldots, k_{n}}^{(1)} & T_{1, k_{3}, \ldots, k_{n}}^{(1)} & T_{0, k_{3}, \ldots, k_{n}}^{(1)} & \cdots \\
\vdots & \vdots & \vdots & \ddots
\end{array}\right)
$$

is said to be Toeplitz matrix of level 2 , denoted as $T_{k_{3}, \ldots, k_{n}}^{(2)}$.

Toeplitz and slant Toeplitz operators 
AJMS

27,1

80

Continuing, we get a block matrix of type

$$
\left(\begin{array}{cccc}
T_{0}^{(n-1)} & T_{-1}^{(n-1)} & T_{-2}^{(n-1)} & \ldots \\
T_{1}^{(n-1)} & T_{0}^{(n-1)} & T_{-1}^{(n-1)} & \ldots \\
T_{2}^{(n-1)} & T_{1}^{(n-1)} & T_{0}^{(n-1)} & \ldots \\
\vdots & \vdots & \vdots & \ddots
\end{array}\right)
$$

called Toeplitz matrix of level $n$ denoted as $T^{(n)}$.

Theorem 3.3. A bounded linear operator $A$ on $H^{2}\left(\mathbb{D}^{n}\right)$ can be represented as a Toeplitz matrix of level $n$ if and only if $\left\langle A e_{k+\epsilon_{j}}, e_{t+\epsilon_{j}}\right\rangle=\left\langle A e_{k}, e_{t}\right\rangle \forall k, t \in Z_{+}^{n}$ and $1 \leq j \leq n$.

Proof is similar to that of Theorem 2.6 and is therefore omitted.

Theorem 3.4. A necessary and sufficient condition that an operator on $H^{2}\left(\mathbb{D}^{n}\right)$ be a Toeplitz operator is that it can be represented as a Toeplitz matrix of level $n$.

Proof. Let $A=T_{\varphi}=P M_{\varphi}$ for $\varphi \in L^{\infty}\left(\mathbb{T}^{n}\right)$. Then for $k, t \in \mathbb{Z}_{+}^{n}$ and $1 \leq j \leq n$, we have

$$
\left\langle A e_{k+\epsilon_{j}}, e_{t+\epsilon_{j}}\right\rangle=\left\langle P M_{\varphi} e_{k+\epsilon_{j}}, e_{t+\epsilon_{j}}\right\rangle=\left\langle M_{\varphi} e_{k+\epsilon_{j}}, e_{t+\epsilon_{j}}\right\rangle
$$

As $M_{\varphi}$ is a Laurent operator, so Corollary 2.9 implies that

$$
\left\langle M_{\varphi} e_{k+\epsilon_{j}}, e_{t+\epsilon_{j}}\right\rangle=\left\langle M_{\varphi} e_{k}, e_{t}\right\rangle
$$

Combining Eqs. (3.1) and (3.2) we get

$$
\left\langle A e_{k+\epsilon_{j}}, e_{t+\epsilon_{j}}\right\rangle=\left\langle M_{\varphi} e_{k}, e_{t}\right\rangle=\left\langle P M_{\varphi} e_{k}, e_{t}\right\rangle=\left\langle A e_{k}, e_{t}\right\rangle .
$$

Thus by Theorem 3.3, $A$ can be represented as a Toeplitz matrix of level $n$.

Conversely, suppose $A$ is a bounded linear operator on $H^{2}\left(\mathbb{D}^{n}\right)$ which can be represented as a Toeplitz matrix of level $n$. Then by Theorem 3.3, we have

$$
\left\langle A e_{k+\epsilon_{j}}, e_{t+\epsilon_{j}}\right\rangle=\left\langle A e_{k}, e_{t}\right\rangle \forall k, t \in \mathbb{Z}_{+}^{n}, 1 \leq j \leq n
$$

For $m \in \mathbb{Z}_{+}$, let $A_{m}: L^{2}\left(\mathbb{T}^{n}\right) \mapsto L^{2}\left(\mathbb{T}^{n}\right)$ be defined as $A_{m}=M_{z^{m}}^{*} A P M_{z^{m}}$ where $z^{m}=$ $z_{1}^{m} \ldots z_{n}^{m}$. Then for $k=\left(k_{1}, \ldots, k_{n}\right)$ and $t=\left(t_{1}, \ldots, t_{n}\right)$ in $\mathbb{Z}_{+}^{n}$, and $m \in \mathbb{Z}_{+}$, repeated application of Eq. (3.3) gives,

$$
\left\langle A_{m} e_{k}, e_{t}\right\rangle=\left\langle A e_{\left(k_{1}+m, \ldots, k_{n}+m\right)}, e_{\left(t_{1}+m, \ldots, t_{n}+m\right)}\right\rangle=\left\langle A e_{k}, e_{t}\right\rangle
$$

For $k, t \in \mathbb{Z}^{n}$, we choose $N \in \mathbb{Z}_{+}$, sufficiently large so that $k_{i}+N, t_{i}+N \in \mathbb{Z}_{+} \forall 1 \leq i \leq n$, and let $\tilde{k}=\left(k_{1}+N, \ldots, k_{n}+N\right), \tilde{t}=\left(t_{1}+N, \ldots, t_{n}+N\right)$. Then by Eq. (3.4), we have $\forall m \in \mathbb{Z}_{+}$,

$$
\left\langle A_{m} e_{\tilde{k}}, e_{\tilde{t}}\right\rangle=\left\langle A e_{\tilde{k}}, e_{\tilde{t}}\right\rangle
$$

Also $e_{\tilde{t}}=M_{z^{N}} e_{t}, e_{\tilde{k}}=M_{z^{N}} e_{k}$ and $P e_{\tilde{k}}=e_{\tilde{k}}$. Using this in Eq. (3.5) we get

$$
\left\langle M_{z^{N}}^{*} A_{m} P M_{z^{N}} e_{k}, e_{t}\right\rangle=\left\langle M_{z^{N}}^{*} A P M_{z^{N}}^{*} e_{k}, e_{t}\right\rangle \Rightarrow\left\langle A_{m+N} e_{k}, e_{t}\right\rangle=\left\langle A_{N} e_{k}, e_{t}\right\rangle
$$

Thus $\forall k, t \in \mathbb{Z}^{n}, \exists N \in \mathbb{Z}_{+}$such that

$$
\left\langle A_{m+N} e_{k}, e_{t}\right\rangle=\left\langle A_{N} e_{k}, e_{t}\right\rangle \forall m \in \mathbb{Z}_{+}
$$


Therefore, if $f$ and $g$ are finite linear combinations of $e_{k}$ for $k \in \mathbb{Z}^{n}$, then the sequence $\left\{\left\langle A_{n} f, g\right\rangle\right\}_{n \in \mathbb{Z}_{+}}$is convergent. Also as $\left\|A_{n}\right\| \leq\|A\| \forall n \in \mathbb{Z}_{+}$, so the sequence $\left\{A_{n}\right\}_{n \in \mathbb{Z}_{+}}$of operators on $L^{2}\left(\mathbb{T}^{n}\right)$ is weakly convergent to a bounded operator $A_{\infty}$ on $L^{2}\left(\mathbb{T}^{n}\right)$.

Claim: $A_{\infty}$ is a Laurent operator.

As in Eq. (3.6), for $k, t \in \mathbb{Z}^{n}$ and $N \in \mathbb{Z}_{+}$, we have

$$
\left\langle A_{m+N} e_{k+\epsilon_{i}}, e_{t+\epsilon_{i}}\right\rangle=\left\langle A_{N} e_{k+\epsilon_{i}}, e_{t+\epsilon_{i}}\right\rangle \forall 1 \leq i \leq n
$$

Taking limit as $m \rightarrow \infty$, in Eqs. (3.6) and (3.7) we get

$$
\begin{aligned}
& \left\langle A_{\infty} e_{k}, e_{t}\right\rangle=\left\langle A_{N} e_{k}, e_{t}\right\rangle=\left\langle M_{z^{N}}^{*} A P M_{z^{N}} e_{k}, e_{t}\right\rangle=\left\langle A e_{\tilde{k}}, e_{\tilde{t}}\right\rangle \text {, and } \\
& \left\langle A_{\infty} e_{k+\epsilon_{i}}, e_{t+\epsilon_{i}}\right\rangle=\left\langle A_{N} e_{k+\epsilon_{i}}, e_{t+\epsilon_{i}}\right\rangle=\left\langle A e_{\tilde{k}+\epsilon_{i}}, e_{\tilde{t}+\epsilon_{i}}\right\rangle=\left\langle A e_{\tilde{k}}, e_{\tilde{t}}\right\rangle .
\end{aligned}
$$

Thus $\left\langle A_{\infty} e_{k}, e_{t}\right\rangle=\left\langle A_{\infty} e_{k+\epsilon_{i}}, e_{t+\epsilon_{i}}\right\rangle \forall 1 \leq i \leq n, k, t \in \mathbb{Z}^{n}$, and hence Corollary 2.9 implies that $A_{\infty}$ is a Laurent operator, and the claim is established.

From Eq. (3.4) we have $\left\langle A_{m} e_{k}, e_{t}\right\rangle=\left\langle A e_{k}, e_{t}\right\rangle \forall k, t \in \mathbb{Z}_{+}^{n}$, and $m \in \mathbb{Z}_{+}$. Thus for $f, g \in H^{2}\left(\mathbb{D}^{n}\right)$, we have $\left\langle A_{m} f, g\right\rangle=\langle A f, g\rangle$.

Therefore $\left\langle P A_{\infty} f, g\right\rangle=\left\langle A_{\infty} f, g\right\rangle=\lim _{m \rightarrow \infty}\left\langle A_{m} f, g\right\rangle=\langle A f, g\rangle \forall f, g \in H^{2}\left(\mathbb{D}^{n}\right)$. This implies that $\left.P A_{\infty}\right|_{H^{2}\left(\mathbb{D}^{n}\right)}=A$, where $A_{\infty}$ is a Laurent operator. Thus, $A$ is a Toeplitz operator.

Definition 3.5. For $1 \leq j \leq n$, we define $U_{z_{j}}: H^{2}\left(\mathbb{D}^{n}\right) \mapsto H^{2}\left(\mathbb{D}^{n}\right)$ as $U_{z_{j}} f(z)=z_{j} f(z)$ $\forall f \in H^{2}\left(\mathbb{D}^{n}\right), z \in \mathbb{T}^{n}$.

Remark 3.6. $U_{z_{j}} e_{k}=e_{k+\epsilon_{j}} \forall k \in \mathbb{Z}_{+}^{n}, 1 \leq j \leq n$.

Theorem 3.7. For $1 \leq j \leq n$ and $k=\left(k_{1}, \ldots, k_{n}\right) \in \mathbb{Z}_{+}^{n}$, we have

$$
U_{z_{j}}^{*} e_{k}=\left\{\begin{array}{cc}
e_{k-\epsilon_{j}} & \text { if } k_{j} \neq 0 \\
0 & \text { Otherwise }
\end{array}\right.
$$

Proof. Let $f \in H^{2}\left(\mathbb{D}^{n}\right)$ where $f(z)=\sum_{m \in \mathbb{Z}_{+}^{n}} f_{m} z^{m}$.

Then for $1 \leq j \leq n$ and $k=\left(k_{1}, \ldots, k_{n}\right) \in \mathbb{Z}_{+}^{n}$, we have

$$
\begin{array}{r}
\left\langle U_{z_{j}} f, e_{k}\right\rangle=\left\langle\sum_{m \in \mathbb{Z}_{+}^{n}} f_{m} z^{m+\epsilon_{j}, z^{k}}\right\rangle=\sum_{m \in \mathbb{Z}_{+}^{n}} f_{m}\left\langle z^{m+\epsilon_{j}, z^{k}}\right\rangle \\
=\left\{\begin{array}{ll}
f_{k-\epsilon_{j}} & \text { if } k_{j} \neq 0 \\
0 & \text { if } k_{j}=0
\end{array}= \begin{cases}\left\langle f, e_{k-\epsilon_{j}}\right\rangle & \text { if } k_{j} \neq 0 \\
0 & \text { if } k_{j}=0\end{cases} \right. \\
\text { Thus, } U_{z_{j}}^{*} e_{k}=\left\{\begin{array}{lll}
e_{k-\epsilon_{j}} & \text { if } k_{j} \neq 0 & \\
0 & \text { Otherwise }
\end{array}\right.
\end{array}
$$

Remark 3.8. For $1 \leq j \leq n$ and $k=\left(k_{1}, \ldots, k_{n}\right) \in \mathbb{Z}_{+}^{n}$, we have

$$
U_{z_{j}}^{*} U_{z_{j}} e_{k}=e_{k} \text { and } U_{z_{j}} U_{z_{j}}^{*} e_{k}= \begin{cases}e_{k} & \text { if } k_{j} \neq 0 \\ 0 & \text { Otherwise }\end{cases}
$$


AJMS

27,1

82

Theorem 3.9. Let $A$ be a bounded linear operator on $H^{2}\left(\mathbb{D}^{n}\right)$. Then $A$ is a Toeplitz operator if and only if $U_{z_{j}}^{*} A U_{z_{j}}=A \forall 1 \leq j \leq n$.

Proof. If $A$ is a Toeplitz operator on $H^{2}\left(\mathbb{D}^{n}\right)$, then, by Theorem 3.4, $A$ can be represented as a Toeplitz matrix of level- $n$. This implies, by Theorem 3.3, that $\left\langle A e_{k+\epsilon_{j}}, e_{t+\epsilon_{j}}\right\rangle=$ $\left\langle A e_{k}, e_{t}\right\rangle \forall k, t \in \mathbb{Z}_{+}^{n}, 1 \leq j \leq n$. Applying Remark 3.6 to this relation we further deduce that $\left\langle U_{z_{j}}^{*} A U_{z_{j}} e_{k}, e_{t}\right\rangle=\left\langle A e_{k}, e_{t}\right\rangle \forall k, t \in \mathbb{Z}_{+}^{n}, 1 \leq j \leq n$, which in turn implies that $U_{z_{j}}^{*} A U_{z_{j}}=A \forall 1 \leq j \leq n$.

Similarly, assuming $U_{z_{j}}^{*} A U_{z_{j}}=A \forall 1 \leq j \leq n$ and applying Theorems 3.3, 3.4 and Remark 3.6 it can be shown that $A$ is a Toeplitz operator on $H^{2}\left(\mathbb{D}^{n}\right)$.

\section{Slant Toeplitz operator on $L^{2}\left(\mathbb{T}^{n}\right)$}

Let us consider a sequence of scalars $\left\{a_{k}\right\}_{k \in \mathbb{Z}^{n}}$. A matrix of the type

$$
\left(\begin{array}{ccccc}
\ddots & \vdots & \vdots & \vdots & \vdots \\
\ldots & a_{\left(-1, k_{2}, \ldots, k_{n}\right)} & a_{\left(-2, k_{2}, \ldots, k_{n}\right)} & a_{\left(-3, k_{2}, \ldots, k_{n}\right)} & \ldots \\
\ldots & a_{\left(1, k_{2}, \ldots, k_{n}\right)} & {\left[a_{\left(0, k_{2}, \ldots, k_{n}\right)}\right]} & a_{\left(-1, k_{2}, \ldots, k_{n}\right)} & \ldots \\
\ldots & a_{\left(3, k_{2}, \ldots, k_{n}\right)} & a_{\left(2, k_{2}, \ldots, k_{n}\right)} & a_{\left(1, k_{2}, \ldots, k_{n}\right)} & \ldots \\
\vdots & \vdots & \vdots & \vdots & \ddots
\end{array}\right)
$$

is said to be slant Toeplitz matrix of level-1, denoted as $A_{k_{2}, \ldots, k_{n}}^{(1)}$. Observe that it is the matrix obtained by eliminating all odd rows of the Laurent matrix of level-1 namely $L_{k_{2}, \ldots, k_{n}}^{(1)}$.

Similarly if we eliminate all odd rows of the block Laurent matrix

$$
\left(\begin{array}{ccccc}
\ddots & \vdots & \vdots & \vdots & \vdots \\
\ldots & A_{0, k_{3}, \ldots, k_{n}}^{(1)} & A_{-1, k_{3}, \ldots, k_{n}}^{(1)} & A_{-2, k_{3}, \ldots, k_{n}}^{(1)} & \ldots \\
\ldots & A_{1, k_{3}, \ldots, k_{n}}^{(1)} & {\left[A_{0, k_{3}, \ldots, k_{n}}^{(1)}\right]} & A_{-1, k_{3}, \ldots, k_{n}}^{(1)} & \ldots \\
\ldots & A_{2, k_{3}, \ldots, k_{n}}^{(1)} & A_{1, k_{3}, \ldots, k_{n}}^{(1)} & A_{0, k_{3}, \ldots, k_{n}}^{(1)} & \ldots \\
\vdots & \vdots & \vdots & \vdots & \ddots
\end{array}\right)
$$

we get the slant Toeplitz matrix of level-2, denotd by $A_{k_{3}, \ldots, k_{n}}^{(2)}$ and given by

$$
\left(\begin{array}{ccccc}
\ddots & \vdots & \vdots & \vdots & \vdots \\
\ldots & A_{-1, k_{3}, \ldots, k_{n}}^{(1)} & A_{-2, k_{3}, \ldots, k_{n}}^{(1)} & A_{-3, k_{3}, \ldots, k_{n}}^{(1)} & \ldots \\
\ldots & A_{1, k_{3}, \ldots, k_{n}}^{(1)} & {\left[A_{0, k_{3}, \ldots, k_{n}}^{(1)}\right]} & A_{-1, k_{3}, \ldots, k_{n}}^{(1)} & \ldots \\
\ldots & A_{3, k_{3}, \ldots, k_{n}}^{(1)} & A_{2, k_{3}, \ldots, k_{n}}^{(1)} & A_{1, k_{3}, \ldots, k_{n}}^{(1)} & \ldots \\
\vdots & \vdots & \vdots & \vdots & \ddots
\end{array}\right)
$$


Continuing in this way, we eliminate the odd rows of the block Laurent matrix

$$
\left(\begin{array}{ccccc}
\ddots & \vdots & \vdots & \vdots & \vdots \\
\ldots & A_{0}^{(n-1)} & A_{-1}^{(n-1)} & A_{-2}^{(n-1)} & \ldots \\
\ldots & A_{1}^{(n-1)} & {\left[A_{0}^{(n-1)}\right]} & A_{-1}^{(n-1)} & \ldots \\
\ldots & A_{2}^{(n-1)} & A_{1}^{(n-1)} & A_{0}^{(n-1)} & \ldots \\
\vdots & \vdots & \vdots & \vdots & \ddots
\end{array}\right)
$$

Toeplitz and slant Toeplitz operators

to get the slant Toeplitz matrix of level- $n$, which is

$$
A^{(n)}=\left(\begin{array}{ccccc}
\ddots & \vdots & \vdots & \vdots & \vdots \\
\ldots & A_{-1}^{(n-1)} & A_{-2}^{(n-1)} & A_{-3}^{(n-1)} & \ldots \\
\ldots & A_{1}^{(n-1)} & {\left[A_{0}^{(n-1)}\right]} & A_{-1}^{(n-1)} & \ldots \\
\ldots & A_{3}^{(n-1)} & A_{2}^{(n-1)} & A_{1}^{(n-1)} & \ldots \\
\vdots & \vdots & \vdots & \vdots & \ddots
\end{array}\right)
$$

Theorem 4.1. If $T$ is a bounded linear operator on $L^{2}\left(\mathbb{T}^{n}\right)$, then $T$ can be represented as $a$ slant Toeplitz matrix of level-n if and only if $\left\langle T e_{k+2 \epsilon_{j}}, e_{t+\epsilon_{j}}\right\rangle=\left\langle T e_{k}, e_{t}\right\rangle \forall k, t \in \mathbb{Z}^{n}$, and $1 \leq j \leq n$.

The proof follows as in Theorem 2.6, and is therefore omitted.

Theorem 4.2. A bounded linear operator $T$ on $L^{2}\left(\mathbb{T}^{n}\right)$ can be represented as a slant Toeplitz matrix of level-n if and only if $M_{z_{j}} T=T M_{z_{j}^{2}} \forall 1 \leq j \leq n$.

Proof. Let $j$ be an integer such that $1 \leq j \leq n$. Then,

$$
M_{z_{j}} T=T M_{z_{j}^{2}} \forall 1 \leq j \leq n
$$

if and only if $\left\langle M_{z_{j}} T e_{k}, e_{t}\right\rangle=\left\langle T M_{z_{j}^{2}} e_{k}, e_{t}\right\rangle, \forall k, t \in \mathbb{Z}^{n}$ and $\forall 1 \leq j \leq n$

if and only if $\left\langle T e_{k}, e_{t-\epsilon_{j}}\right\rangle=\left\langle T e_{k+2 \epsilon_{j}}, e_{t}\right\rangle, \forall k, t \in \mathbb{Z}^{n}$ and $\forall 1 \leq j \leq n$ if and only if $\left\langle T e_{k}, e_{t}\right\rangle=\left\langle T e_{k+2 \epsilon_{j}}, e_{t+\epsilon_{j}}\right\rangle, \forall k, t \in \mathbb{Z}^{n}$ and $\forall 1 \leq j \leq n$ The result now follows from Theorem 4.1.

Definition 4.3. $W: L^{2}\left(\mathbb{T}^{n}\right) \mapsto L^{2}\left(\mathbb{T}^{n}\right)$ is defined to be the linear operator such that for each $k \in \mathbb{Z}^{n}, W e_{k}=\left\{\begin{array}{cc}e_{\frac{k}{2}}, & \text { if } k \text { is even; } \\ 0, & \text { if } k \text { is odd. }\end{array}\right.$

So for $f(z)=\sum_{j \in \mathbb{Z}^{n}} a_{j} z^{j} \in L^{2}\left(\mathbb{T}^{n}\right)$, we have $W f(z)=\sum_{j \in \mathbb{Z}^{n}} a_{2 j} z^{j}$.

\section{Remark 4.4.}

(i) For $k, t \in \mathbb{Z}^{n}$

$$
\left\langle W e_{k}, e_{t}\right\rangle=\left\{\begin{array}{cc}
1 & \text { if } 2 t=k \\
0 & \text { Otherwise }
\end{array}\right.
$$

and so $\|W\|=1$ 
AJMS

27,1

(ii) $W^{*} e_{t}=e_{2 t} \forall t \in \mathbb{Z}^{n}$, because $\left\langle W e_{k}, e_{t}\right\rangle=\left\langle e_{k}, e_{2 t}\right\rangle \forall k, t \in \mathbb{Z}^{n}$. So for $f(z)=\sum_{p \in \mathbb{Z}^{n}} a_{p} z^{p}$ $\in L^{2}\left(\mathbb{T}^{n}\right)$, we have $W^{*} f(z)=\sum_{p \in Z^{n}} a_{p} z^{2 p}=f\left(z^{2}\right)$.

Definition 4.5. For $\varphi \in L^{\infty}\left(\mathbb{T}^{n}\right)$, we define the slant Toeplitz operator $A_{\varphi}$ as $W M_{\varphi}$. Hence, if $\varphi$ is the constant function 1 then $W=A_{1}$.

84

Theorem 4.6. $\left\|A_{\varphi}\right\| \leq\|\varphi\|_{\infty}$.

Proof. $\left\|A_{\varphi}\right\|=\left\|W M_{\varphi}\right\| \leq\|W\|\left\|M_{\varphi}\right\|=\left\|M_{\varphi}\right\|=\|\varphi\|_{\infty}$.

Theorem 4.7. If $A_{\varphi}$ is a slant Toeplitz operator on $L^{2}\left(\mathbb{T}^{n}\right)$, then

$$
\left\langle A_{\varphi} e_{k+2 \epsilon_{j}}, e_{t+\epsilon_{j}}\right\rangle=\left\langle A_{\varphi} e_{k}, e_{t}\right\rangle \forall k, t \in \mathbb{Z}^{n}, 1 \leq j \leq n .
$$

Proof. Since $A_{\varphi}=W M_{\varphi}$, the result follows immediately from Corollary 2.9 and Remark 4.4(ii).

Theorem 4.8. A bounded linear operator A on $L^{2}\left(\mathbb{T}^{n}\right)$ is a slant Toeplitz operator if and only if A can be represented as a slant Toeplitz matrix of level-n.

If $A$ is a slant Toeplitz operator then by Theorems 4.1 and 4.7 it follows immediately that $A$ can be represented as a slant Toeplitz matrix of level- $n$. However, for the converse implication we defer the proof to Section 6 as we first have to establish a few more results needed thereof.

\section{Properties of $W$ and $W^{*}$}

Definition 5.1. Let $P_{e}$ be the projection of $L^{2}\left(\mathbb{T}^{n}\right)$ onto the closed span of $\left\{e_{2 k}\right\}_{k \in Z^{n}}$ in $L^{2}\left(\mathbb{T}^{n}\right)$. Thus for $j \in \mathbb{Z}^{n}$ we have $P_{e} e_{j}= \begin{cases}e_{j}, & \text { if } j \text { is even; } \\ 0, & \text { otherwise. }\end{cases}$

Or equivalently we have $P_{e}\left(\sum_{k \in Z^{n}} a_{k} z^{k}\right)=\sum_{k \in Z^{n}} a_{2 k} z^{2 k}$.

We make the following observations:

(i) $W W^{*}=I$, and so $W$ is a co-isometry on $L^{2}\left(\mathbb{T}^{n}\right)$.

(ii) $W^{*} W=P_{e}$, and so $W$ is an isometry on $P_{e}\left(L^{2}\left(\mathbb{T}^{n}\right)\right)$.

In the following few results we refer to the subset $S$ of $\mathbb{Z}^{n}$, the definition for which was already given in Section 2.

Lemma 5.2. Let $S=\left\{\left(t_{1}, \ldots, t_{n}\right) \in \mathbb{Z}^{n} \mid\right.$ each $t_{i}$ is either 0 or 1$\}$. Then for $k \in \mathbb{Z}^{n}, k$ odd, there exist unique $\tilde{k} \in \mathbb{Z}^{n}$ and $0 \neq t \in S$ such that $k=2 \tilde{k}+t$.

Proof. Let $k=\left(k_{1}, \ldots, k_{n}\right) \in \mathbb{Z}^{n}$. For each $1 \leq i \leq n$ there exists $\tilde{k}_{i} \in \mathbb{Z}$ such that

$$
k_{i}= \begin{cases}2 \tilde{k}_{i} & \text { if } k_{i} \text { is even, } \\ 2 \tilde{k}_{i}+1 & \text { if } k_{i} \text { is odd }\end{cases}
$$

Let $\tilde{k}=\left(\tilde{k}_{1}, \ldots, \tilde{k}_{n}\right)$ and $t=\left(t_{1}, \ldots, t_{n}\right)$ where

$$
t_{i}= \begin{cases}0 & \text { if } k_{i} \text { is even, } \\ 1 & \text { if } k_{i} \text { is odd }\end{cases}
$$

Then $k=2 \tilde{k}+t$ where $\tilde{k} \in \mathbb{Z}^{n}, t \in S$. Also $k$ is odd implies $t_{i}=1$ for at least one $i$, so that $t$ is non-zero. 
Uniqueness Suppose $\exists k^{\prime}=\left(k_{1}^{\prime}, \ldots, k_{n}^{\prime}\right) \in \mathbb{Z}^{n}$ and $t^{\prime}=\left(t_{1}^{\prime}, \ldots, t_{n}^{\prime}\right) \in S$ such that $k=2 k^{\prime}+t^{\prime}$.

Let if possible $t^{\prime} \neq t$. Without loss of generality we suppose $t_{1}^{\prime} \neq t_{1}$, so that either $t_{1}^{\prime}=1$ and $t_{1}=0$, or $t_{1}^{\prime}=0$ and $t_{1}=1$.

Now $t_{1}^{\prime}=1$ implies $k_{1}=2 k_{1}^{\prime}+t_{1}^{\prime}=2 k_{1}^{\prime}+1$, which is odd,

and $t_{1}=0$ implies $k_{1}=2 \tilde{k}_{1}+t_{1}=2 \tilde{k}_{1}$, which is even. Thus we get a contradiction.

Similarly, $t_{1}^{\prime}=0$ and $t_{1}=1$ give us a contradiction.

Thus $t^{\prime}=t$ implies $2 k^{\prime}=2 \tilde{k}$ or $k^{\prime}=\tilde{k}$.

Remark 5.3. Every non zero entry in $S$ is odd. Also $\epsilon_{j} \in S$ for $j=1,2, \ldots, n$.

Lemma 5.4. For $t, k \in S$ with $t \neq 0, k \neq 0$, we have $t+k$ is even if and only if $t=k$.

Proof. Suppose $t=k$. Then $t+k=2 t$, which is even.

Conversely, suppose $t \neq k$. Then without loss of generality we assume that $t_{1} \neq k_{1}$, where $t=\left(t_{1}, \ldots, t_{n}\right), k=\left(k_{1}, \ldots, k_{n}\right)$. Thus, either $t_{1}=0$ and $k_{1}=1$; or $t_{1}=1$ and $k_{1}=0$. In any case,

$t+k=\left(t_{1}+k_{1}, \ldots, t_{n}+k_{n}\right)$, where $t_{1}+k_{1}=1$, and so $t+k$ is odd.

Thus $t \neq k$ implies that $t+k$ odd, or equivalently, $t+k$ even implies that $t=k$.

Theorem 5.5. $W M_{z^{2 k}} W^{*}=M_{z^{k}}$ if $k \in \mathbb{Z}^{n}$, and $W M_{z^{t}} W^{*}=0$ if $t \in \mathbb{Z}^{n}$ is odd.

Proof. For $k, p \in \mathbb{Z}^{n}, W M_{z^{2 k}} W^{*} e_{p}=W M_{z^{2 k}} e_{2 p}=W e_{2(p+k)}=e_{(p+k)}=M_{z^{k}} e_{p}$. Now if $t \in \mathbb{Z}^{n}$ is odd, then by Lemma $5.2,2 p+t$ is odd, and so we have $W M_{z^{t}} W^{*} e_{p}=$ $W M_{z^{t}} e_{2 p}=W e_{(2 p+t)}=0$.

Corollary 5.6. $W M_{z_{j}} W^{*}=0 \forall 1 \leq j \leq n$.

Proof. This follows from Theorem 5.5. Taking $t=\epsilon_{j}$ we get $z^{t}=z_{j}$ where $t$ is odd. Thus $W M_{z_{j}}-W^{*}=0$.

Corollary 5.7. $W M_{z} W^{*}=0$

Proof. Let $t=\epsilon_{1}+\epsilon_{2}+\cdots+\epsilon_{n}$. Then $t$ is odd and $z^{t}=z_{1} \cdots z_{n}=z$. So $W M_{z} W^{*}=0$.

Theorem 5.8. Let $f, g \in L^{2}\left(\mathbb{T}^{n}\right)$ such that $f g \in L^{2}\left(\mathbb{T}^{n}\right)$. Then

(i) $W^{*}(f g)=\left(W^{*} f\right)\left(W^{*} g\right)$,

(ii) $W\left(\left(W^{*} f\right)\left(W^{*} g\right)\right)=f g$.

Proof. (i) $W^{*}\left(e_{k} e_{t}\right)=W^{*} e_{(k+t)}=e_{2(k+t)}=e_{2 k} e_{2 t}=\left(W^{*} e_{k}\right)\left(W^{*} e_{t}\right)$ for $k, t \in \mathbb{Z}^{n}$. Thus for $f, g \in L^{2}\left(\mathbb{T}^{n}\right), W^{*}(f g)=\left(W^{*} f\right)\left(W^{*} g\right)$, provided $f g \in L^{2}\left(\mathbb{T}^{n}\right)$.

(ii) As $W W^{*}=I$ so the result follows immediately from (i).

Theorem 5.9. For $\varphi \in L^{\infty}\left(\mathbb{T}^{n}\right), W A_{\varphi}^{*}=M_{\psi}$ where $\psi=W \bar{\varphi}$.

Proof. Let $\varphi(z)=\sum_{t \in \mathbb{Z}^{n}} a_{t} z^{t}$, so that $\bar{\varphi}(z)=\sum_{t \in \mathbb{Z}^{n}} \bar{a}_{t} z^{-t}$. Then $\psi(z)=W \bar{\varphi}(z)=\sum_{t \in Z^{n}}$ $\bar{a}_{t} W z^{-t}=\sum_{t \in \mathbb{Z}^{n}} \bar{a}_{2 t} z^{-t}$ and $M_{\psi}=\sum_{t \in \mathbb{Z}^{n}} \bar{a}_{2 t} M_{z^{-t}}$.

Again, $A_{\varphi}=W M_{\varphi}$ implies $A_{\varphi}^{*}=M_{\varphi}^{*} W^{*}=M_{\bar{\varphi}} W^{*}=\sum_{t \in \mathbb{Z}^{n}} \bar{a}_{t} M_{z^{-t}} W^{*}$.

Hence by Theorem 5.5,

$$
W A_{\varphi}^{*}=\sum_{t \in \mathbb{Z}^{n}} \bar{a}_{t} W M_{z^{-t}} W^{*}=\sum_{t \in \mathbb{Z}^{n}} \bar{a}_{2 t} M_{z^{-t}}=M_{\psi} .
$$

Corollary 5.10. If $\varphi \in L^{\infty}\left(\mathbb{T}^{n}\right)$, then $W \varphi \in L^{\infty}\left(\mathbb{T}^{n}\right)$.

Proof. Let $\varphi \in L^{\infty}\left(\mathbb{T}^{n}\right)$. Then by Theorem 5.9, $M_{W \varphi}=W A_{\bar{\varphi}}^{*}$, which is bounded on $L^{2}\left(\mathbb{T}^{n}\right)$. Therefore $W \varphi \in L^{\infty}\left(\mathbb{T}^{n}\right)$.
Toeplitz and slant Toeplitz operators 
AJMS

27,1
Lemma 5.11. Let $f \in L^{2}\left(\mathbb{T}^{n}\right)$ and $f(z)=\sum_{k \in Z^{n}} a_{k} z^{k}$. If for $t \in S$, we define $f_{t}(z)=$ $\sum_{k \in \mathbb{Z}^{n}} a_{2 k+t} z^{k}$, then $f(z)=\sum_{t \in S} z^{t} f_{t}\left(z^{2}\right)$. Also $f_{0}\left(z^{2}\right)=P_{e} f(z)$ and $f_{0}(z)=W f(z)$.

Proof. Let $f(z)=\sum_{k \in \mathbb{Z}^{n}} a_{k} z^{k}$. If $g(z):=\sum_{k \in \mathbb{Z}^{n}, k \text { even }} a_{k} z^{k}$ and $h(z):=\sum_{k \in \mathbb{Z}^{n}, k \text { odd }} a_{k} z^{k}$, then $f(z)=g(z)+h(z)$. For $t \in S$ define $f_{t}(z)=\sum_{k \in \mathbb{Z}^{n}} a_{2 k+t} z^{k}$. As $f \in L^{2}\left(\mathbb{T}^{n}\right)$, so $f_{t} \in$ $L^{2}\left(\mathbb{T}^{n}\right) \forall t \in S$. We have

$$
g(z)=\sum_{k \in \mathbb{Z}^{n}, k \text { even }} a_{k} z^{k}=\sum_{k \in \mathbb{Z}^{n}} a_{2 k} z^{2 k}=f_{0}\left(z^{2}\right) .
$$

Again, by Lemma 5.2,

$h(z)=\sum_{0 \neq t \in S} \sum_{k \in \mathbb{Z}^{n}} a_{2 k+t} z^{2 k+t}=\sum_{0 \neq t \in S} z^{t}\left(\sum_{k \in \mathbb{Z}^{n}} a_{2 k+t}\left(z^{2}\right)^{k}\right)=\sum_{0 \neq t \in S} z^{t} f_{t}\left(z^{2}\right)$. Thus we have, $f(z)=f_{0}\left(z^{2}\right)+\sum_{0 \neq t \in S} z^{t} f_{t}\left(z^{2}\right)=\sum_{t \in S} z^{t} f_{t}\left(z^{2}\right)$.

Moreover, by Definition 4.3, we have $W g(z)=\sum_{k \in \mathbb{Z}^{n}} a_{2 k} z^{k}=f_{0}(z)$, and $W h(z)=0$, so that $W f(z)=f_{0}(z)$.

Again, by Definition 5.1, we have $P_{e} g(z)=g(z)$ and $P_{e} h(z)=0$ so that $P_{e} f(z)=$ $P_{e} g(z)=g(z)=f_{0}\left(z^{2}\right)$.

Theorem 5.12. Let $f, g \in L^{2}\left(\mathbb{T}^{n}\right)$ such that one of $f$ and $g$ is in $L^{\infty}\left(\mathbb{T}^{n}\right)$. Then $W(f g)=$ $(W f)(W g)+\sum_{0 \neq t \in S} z^{t}\left(W \bar{z}^{t} f\right)\left(W \bar{z}^{t} g\right)$.

Proof. By Lemma 5.11, we have, $f(z)=f_{0}\left(z^{2}\right)+\sum_{0 \neq t \in S} z^{t} f_{t}\left(z^{2}\right)$ and $g(z)=g_{0}\left(z^{2}\right)+$ $\sum_{0 \neq t \in S} z^{t} g_{t}\left(z^{2}\right)$, where $f_{0}\left(z^{2}\right)=P_{e} f(z)$, and $g_{0}\left(z^{2}\right)=P_{e} g(z)$. Hence,

$$
\begin{array}{r}
f g=f_{0}\left(z^{2}\right) g_{0}\left(z^{2}\right)+f_{0}\left(z^{2}\right)\left(\sum_{0 \neq t \in S} z^{t} g_{t}\left(z^{2}\right)\right)+g_{0}\left(z^{2}\right)\left(\sum_{0 \neq t \in S} z^{t} f_{t}\left(z^{2}\right)\right) \\
+\left(\sum_{0 \neq t \in S} z^{t} f_{t}\left(z^{2}\right)\right)\left(\sum_{0 \neq t \in S} z^{t} g_{t}\left(z^{2}\right)\right)
\end{array}
$$

Applying Remark 4.4(ii), Theorem 5.8(ii) and Lemma 5.11 in that order, we get,

$$
W\left(f_{0}\left(z^{2}\right) g_{0}\left(z^{2}\right)\right)=W\left(W^{*} f_{0}(z) \cdot W^{*} g_{0}(z)\right)=f_{0}(z) g_{0}(z)=(W f(z))(W g(z))
$$

As $f_{0}\left(z^{2}\right)\left(\sum_{0 \neq t \in S} z^{t} g_{t}\left(z^{2}\right)\right)$ and $g_{0}\left(z^{2}\right)\left(\sum_{0 \neq t \in S} z^{t} f_{t}\left(z^{2}\right)\right)$ are expressions that involve only odd powers of $z$, so by Definition 4.3,

$$
W\left[f_{0}\left(z^{2}\right) \sum_{t \in S, t \neq 0} z^{t} g_{t}\left(z^{2}\right)\right]=0 \text { and } W\left[g_{0}\left(z^{2}\right) \sum_{t \in S, t \neq 0} z^{t} f_{t}\left(z^{2}\right)\right]=0 .
$$

Again, $\left(\sum_{0 \neq t \in S} z^{t} g_{t}\left(z^{2}\right)\right)\left(\sum_{0 \neq t \in S} z^{t} f_{t}\left(z^{2}\right)\right)=\sum_{0 \neq t \in S} \sum_{0 \neq t \in S} z^{t+k} g_{t}\left(z^{2}\right) f_{k}\left(z^{2}\right)$, where $t+k$ is even iff $t=k$, by Lemma 5.4. Therefore by Definition 4.3,

$$
\begin{aligned}
W\left(\sum_{0 \neq t \in S} z^{t} g_{t}\left(z^{2}\right)\right)\left(\sum_{0 \neq t \in S} z^{t} f_{t}\left(z^{2}\right)\right) & =\sum_{0 \neq t \in S} W\left(z^{2 t}\right) W\left(g_{t}\left(z^{2}\right)\right) W\left(f_{t}\left(z^{2}\right)\right) \\
& =\sum_{0 \neq t \in S} z^{t} W\left(g_{t}\left(z^{2}\right)\right) W\left(f_{t}\left(z^{2}\right)\right) .
\end{aligned}
$$

As $g(z)=\sum_{k \in S} z^{k} g_{k}\left(z^{2}\right)$, so for any $0 \neq t \in S$, we have 


$$
\begin{gathered}
z^{t} g_{t}\left(z^{2}\right)=g(z)-\sum_{k \in S, k \neq t} z^{k} g_{k}\left(z^{2}\right) \Rightarrow g_{t}\left(z^{2}\right)=\bar{z}^{t} g(z)-\sum_{k \in S, k \neq t} z^{k-t} g_{k}\left(z^{2}\right) \text {. Therefore, } \\
W\left(g_{t}\left(z^{2}\right)\right)=W\left(\bar{z}^{t} g(z)\right)
\end{gathered}
$$

operators

Similarly,

$$
W\left(f_{t}\left(z^{2}\right)\right)=W\left(\bar{z}^{t} f(z)\right) .
$$

So, Eqs. (5.1)-(5.6) together imply,

$$
W(f g)=(W f)(W g)+\sum_{r \in S, t \neq 0} z^{t}\left(W \bar{z}^{t} f\right)\left(W \bar{z}^{t} g\right) .
$$

Remark 5.13. The above relation is correct provided the expression on the RHS is in $L^{2}\left(\mathbb{T}^{n}\right)$ i.e. $(W f)(W g)$ and $\left(W \bar{z}^{t} f\right)\left(W \bar{z}^{t} g\right)$ should be $L^{2}\left(\mathbb{T}^{n}\right)$ functions. This is guaranteed by Corollary 5.10 .

\section{Properties of $A_{\varphi}$ and $A_{\varphi}^{*}$}

Lemma 6.1. For a bounded linear operator $A$ on $L^{2}\left(\mathbb{T}^{n}\right)$, we have $M_{z_{j}} A=A M_{z_{j}^{2}} \forall 1 \leq j \leq n$ if and only if $M_{z^{k}} A=A M_{z^{2 k}} \forall k \in \mathbb{Z}^{n}$.

Proof. Suppose $M_{z^{k}} A=A M_{z^{2 k}} \forall k \in \mathbb{Z}^{n}$. In this expression if we put $k=\epsilon_{j}$ for any integer $j$ with $1 \leq j \leq n$, we get $M_{z_{j}} A=A M_{z_{j}^{2}}$. Conversely, if $M_{z_{j}}=A M_{z_{j}^{2}} \forall 1 \leq j \leq n$, then for $k=\left(k_{1}, \ldots, k_{n}\right) \in \mathbb{Z}^{n}$ we have $M_{z^{k}} A=M_{z_{1}}^{k_{1}} \ldots M_{z_{1}}^{k_{n}} A=A M_{z_{1}}^{2 k_{1}} \ldots M_{z_{n}}^{2 k_{n}}=M_{z^{2 k}}$.

Theorem 6.2. A bounded linear operator $A$ on $L^{2}\left(\mathbb{T}^{n}\right)$ is a slant Toeplitz operator if and only if $M_{z^{k}} A=A M_{z^{2 k}} \forall k \in \mathbb{Z}^{n}$. In such a case we have $A=A_{\varphi}$ with $\varphi(z)=\sum_{t \in S} \bar{z}^{t}\left(A e_{t}\right)\left(z^{2}\right)$.

Proof. If $A$ is a slant Toeplitz operator, then the result follows from Theorems 4.8, 4.2 and Lemma 6.1.

Conversely, let $A$ be a bounded linear operator on $L^{2}\left(\mathbb{T}^{n}\right)$ and suppose $M_{z^{k}} A=$ $A M_{z^{2 k}} \forall k \in \mathbb{Z}^{n}$.

We prove the result in the following steps:

(i) For $t \in S$, define $\varphi_{t}(z)=\bar{z}^{t}\left(A e_{t}\right)\left(z^{2}\right)$. We will show that $\varphi_{t} \in L^{\infty}\left(\mathbb{T}^{n}\right)$.

(ii) Define $\varphi(z)=\sum_{t \in S} \varphi_{t}(z)$. We will show that $A=A_{\varphi}$.

Proof of step (i): Let $h \in L^{2}\left(\mathbb{T}^{n}\right)$ with $h(z)=\sum_{k \in \mathbb{Z}^{n}} \delta_{k} z^{k}$. If $\xi(z):=h\left(z^{2}\right)$, then $\xi \in L^{2}\left(\mathbb{T}^{n}\right)$, $\xi(z)=\sum_{k \in \mathbb{Z}^{n}} \delta_{k} z^{2 k}$ and $\|\xi\|=\|h\|$. For $t \in S$ we have

$$
\begin{aligned}
A\left(z^{t} \xi(z)\right) & =A\left(z^{t} \sum_{k \in \mathbb{Z}^{n}} \delta_{k} z^{2 k}\right) \\
& =\sum_{k \in \mathbb{Z}^{n}} \delta_{k} A M_{z^{2 k}} e_{t}(z)=\sum_{k \in \mathbb{Z}^{n}} \delta_{k} M_{z^{k}} A e_{t}(z) \\
& =\left(\sum_{k \in \mathbb{Z}^{n}} \delta_{k} z^{k}\right) A e_{t}(z)=h(z) \cdot A e_{t}(z)=\left(h \cdot A e_{t}\right)(z)
\end{aligned}
$$

Thus, $A M_{z}^{t} \xi=h . A e_{t}$ and $A\left(z^{t} h\left(z^{2}\right)\right)=\left(h . A e_{t}\right)(z) \forall t \in S$

Also, $\left\|M_{A e_{t}} \cdot h\right\|=\left\|A e_{t} \cdot h\right\|=\left\|A M_{z}^{t} \cdot \xi\right\| \leq\|A\|\left\|M_{z}\right\|^{t}\|\xi\|=\|A\|\|h\|$

Therefore $M_{A e_{t}}$ is bounded and so $A e_{t} \in L^{\infty}\left(\mathbb{T}^{n}\right) \forall t \in S$. 
AJMS

27,1

88

This implies that $\varphi_{t} \in L^{\infty}\left(\mathbb{T}^{n}\right) \forall t \in S$.

Proof of step (ii): As $\varphi_{t} \in L^{\infty}\left(\mathbb{T}^{n}\right) \forall t \in S$, so $\varphi \in L^{\infty}\left(\mathbb{T}^{n}\right)$. Let $F \in L^{2}\left(\mathbb{T}^{n}\right)$. So by Lemma 5.11, $F(z)=F_{0}\left(z^{2}\right)+\sum_{0 \neq t \in S} z^{t} F_{t}\left(z^{2}\right)$, where $W F(z)=F_{0}(z)$. Again applying Theorem 5.12 we get

$$
\begin{aligned}
A_{\varphi} F(z) & =W M_{\varphi} F(z)=W(\varphi(z) F(z)) \\
& =(W \varphi(z))(W F(z))+\sum_{0 \neq t \in S} z^{t}\left(W \bar{z}^{t} \varphi(z)\right)\left(W \bar{z}^{t} F(z)\right)
\end{aligned}
$$

By Definition 4.3, we have $W \varphi_{t}(z)=0$ for $0 \neq t \in S$, and so

$$
W \varphi(z)=W \varphi_{0}(z)=W\left(A e_{0}\left(z^{2}\right)\right)=A e_{0}(z) .
$$

Also, by applying Lemma 5.4 we get

$$
\begin{aligned}
\left(W \bar{z}^{t} \varphi(z)\right)\left(W \bar{z}^{t} F(z)\right) & =\left(\sum_{k \in S} W \bar{z}^{k+t}\left(A e_{k}\right)\left(z^{2}\right)\right)\left(\sum_{k \in S} W z^{k-t} F_{k}\left(z^{2}\right)\right) \\
& =\left(W \bar{z}^{2 t} A e_{t}\left(z^{2}\right)\right)\left(W F_{t}\left(z^{2}\right)\right)=\bar{z}^{t} A e_{t}(z) F_{t}(z) . \\
\text { Therefore, } A_{\varphi} F(z) & =A e_{0}(z) F_{0}(z)+\sum_{0 \neq t \in S}|z|^{2 t} A e_{t}(z) F_{t}(z) \\
& =\left(F_{0} . A e_{0}\right)(z)+\sum_{0 \neq t \in S}\left(F_{t} . A e_{t}\right)(z) \\
& =A\left(F_{0}\left(z^{2}\right)\right)+\sum_{0 \neq t \in S} A\left(z^{t} F_{t}\left(z^{2}\right)\right), \quad \text { by Eq. }(6.1) \\
& =A\left[F_{0}\left(z^{2}\right)+\sum_{0 \neq t \in S} z^{t} F_{t}\left(z^{2}\right)\right]=A F(z)
\end{aligned}
$$

Thus, $A_{\varphi} F=A F \forall F \in L^{2}\left(\mathbb{T}^{n}\right)$ which implies that $A_{\varphi}=A$.

We are now in a position to complete the proof of Theorem 4.8, stated in Section 4.

Proof of Theorem 4.8. Suppose $A$ is a bounded linear operator on $L^{2}\left(\mathbb{T}^{n}\right)$ such that $A$ can berepresented as a slant Toeplitz matrix of level- $n$. Then by Theorem 4.2 we have $M_{z_{j}} A=A M_{z_{j}^{2}} \forall 1 \leq j \leq n$. This together with Lemma 6.1 implies that $M_{z^{k}} A=A M_{z^{2 k}} \forall k \in \mathbb{Z}^{n}$. Hence, applying Theorem 6.2, we conclude that $A$ is a slant Toeplitz operator.

Theorem 6.3. $A_{\varphi} A_{\varphi}^{*}=M_{\psi}$ where $\psi=W\left(|\varphi|^{2}\right)$.

Proof. $A_{\varphi} A_{\varphi}^{*}=W M_{\varphi} M_{\varphi}^{*} W^{*}=W M_{|\varphi|^{2}} W^{*}=W\left(W M_{|\varphi|^{2}}\right)^{*}=W A_{|\varphi|^{2}}^{*}=M_{\psi}$, where $\psi=W\left(|\varphi|^{2}\right)$, by Theorem 5.9.

Corollary 6.4. $\left\|A_{\varphi}\right\|^{2}=\|\psi\|_{\infty}$, where $\psi=W\left(|\varphi|^{2}\right)$.

Proof. $\left\|A_{\varphi}\right\|^{2}=\left\|A_{\varphi} A_{\varphi}^{*}\right\|=\left\|M_{\psi}\right\|=\|\psi\|_{\infty}$.

Corollary 6.5. $A_{\varphi}^{*}$ is an isometry iff $W|\varphi|^{2}=1$.

Theorem 6.6. The map $\tau: L^{\infty}\left(T^{n}\right) \mapsto \mathcal{B}\left(L^{2}\left(\mathbb{T}^{n}\right)\right)$ defined as $\tau(\varphi)=A_{\varphi}$ is linear and injective. Here $\mathcal{B}\left(L^{2}\left(\mathbb{T}^{n}\right)\right.$ denotes the algebra of all bounded linear operators on $L^{2}\left(\mathbb{T}^{n}\right)$.

Proof. For $\varphi, \psi \in L^{\infty}\left(\mathbb{T}^{n}\right)$ and $\lambda \in \mathbb{C}$,

$\tau(\lambda \varphi+\psi)=A_{\lambda \varphi+\psi}=W M_{\lambda \varphi+\psi}=\lambda\left(W M_{\varphi}\right)+\left(W M_{\psi}\right)=\lambda A_{\varphi}+A_{\psi}=\lambda \tau(\varphi)+\tau(\psi)$.

Therefore $\tau$ is linear. 
Next to show $\tau$ is injective: Suppose $\tau(\varphi)=0$. This implies $A_{\varphi}=W M_{\varphi}=0$. Let $\varphi(z)=$ $\sum_{k \in \mathbb{Z}^{n}} a_{k} z^{k}$. Then by Lemma 5.11, we have $\varphi(z)=\sum_{\xi \in S} z^{\xi} \varphi_{\xi}\left(z^{2}\right)$ where $\varphi_{\xi}(z)=\sum_{k \in \mathbb{Z}^{n}} a_{2 k+\xi} z^{k}$. So for $t \in S$ we have $\varphi(z) z^{t}=\sum_{\xi \in S} z^{\xi+t} \varphi_{\xi}\left(z^{2}\right)$, and since by Lemma $5.4, \xi+t$ is even only for $\xi=t$, so $W\left(z^{\xi+t} \varphi_{\xi}\left(z^{2}\right)\right)=0$ if $\xi \neq t$. Hence we have

$$
\begin{aligned}
W M_{\varphi} e_{t}(z)=W\left(\varphi(z) z^{t}\right) & =W\left(z^{2 t} \varphi_{t}\left(z^{2}\right)\right), \\
& =W\left(\sum_{k \in \mathbb{Z}^{n}} a_{2 k+t} z^{2(k+t)}\right)=z^{t} \sum_{k \in \mathbb{Z}^{n}} a_{2 k+t} z^{k}=z^{t} \varphi_{t}(z)
\end{aligned}
$$

Therefore, $W M_{\varphi}=0$ implies that $\varphi_{t}(z)=0 \forall t \in S$, from which we can conclude that $\varphi(z)=0$.

Theorem 6.7. $M_{\varphi} A_{\psi}$ is a slant Toeplitz operator and $M_{\varphi} A_{\psi}=A_{\varphi\left(z^{2}\right) . \psi(z)}$.

Proof. For each $k \in \mathbb{Z}^{n}$ we have $M_{z^{k}}\left(M_{\varphi} A_{\psi}\right)=M_{\varphi} M_{z^{k}} A_{\psi}=\left(M_{\varphi} A_{\psi}\right) M_{z^{2 k}}$. So, by Theorem 6.2, $M_{\varphi} A_{\psi}$ is a slant Toeplitz operator. Again, as $A_{\psi}$ is slant Toeplitz, so $M_{z^{k}} A_{\psi}=A_{\psi} M_{z^{2 k}} \forall k \in \mathbb{Z}^{n}$ which implies that $M_{\varphi(z)} A_{\psi}=A_{\psi} M_{\varphi\left(z^{2}\right)} \forall \varphi \in L^{\infty}\left(\mathbb{T}^{n}\right)$.

Therefore, $M_{\varphi} A_{\psi}=A_{\psi} M_{\varphi\left(z^{2}\right)}=W M_{\psi} M_{\varphi\left(z^{2}\right)}=W M_{\psi(z) \varphi\left(z^{2}\right)}=A_{\psi(z) \varphi\left(z^{2}\right)}$.

Theorem 6.8. $A_{\psi} M_{\varphi}=M_{\varphi} A_{\psi}$ if and only if $\psi(z) \varphi(z)=\psi(z) \varphi\left(z^{2}\right)$ for any $z \in \mathbb{T}^{n}$. In particular, if $\psi$ is invertible, then $A_{\psi} M_{\varphi}=M_{\varphi} A_{\psi}$ if and only if $\varphi(z)=$ constant.

Proof. $A_{\psi} M_{\varphi}=W M_{\psi} M_{\varphi}=W M_{\psi \varphi}=A_{\psi \varphi}$, and by Theorem 6.7, $M_{\varphi} A_{\psi}=A_{\varphi\left(z^{2}\right) . \psi(z)}$. Thus $A_{\psi} M_{\varphi}=M_{\varphi} A_{\psi}$ if and only if $A_{\psi(z) \varphi(z)}=A_{\varphi\left(z^{2}\right) \mid \psi(z)}$. In other words, $A_{\psi} M_{\varphi}=M_{\varphi} A_{\psi}$ if and only if $\psi(z) \varphi(z)=\psi(z) \varphi\left(z^{2}\right)$ for any $z \in \mathbb{T}^{n}$.

If $\psi$ is invertible, then there exists $\psi^{-1} \in L^{\infty}\left(\mathbb{T}^{n}\right)$ such that $\psi \psi^{-1}=I=\psi^{-1} \psi$, so that $\varphi(z) \psi(z)=\varphi\left(z^{2}\right) \psi(z)$ if and only if $\varphi(z)=\varphi\left(z^{2}\right)$.

Now if $\varphi(z)=\sum_{k \in \mathbb{Z}^{n}} a_{k} z^{k}$, then $\varphi\left(z^{2}\right)=\sum_{k \in \mathbb{Z}^{n}} a_{k} z^{2 k}$, and $\operatorname{so} \varphi(z)=\varphi\left(z^{2}\right)$ implies $\sum_{k \in \mathbb{Z}^{n}, k \text { odd }} a_{k} z^{k}+\sum_{0 \neq k \in \mathbb{Z}^{n}}\left(a_{2 k}-a_{k}\right) z^{2 k}=0$

This gives $a_{k}=0 \forall k$ odd and $a_{2 k}=a_{k} \forall 0 \neq k \in \mathbb{Z}^{n}$

Therefore $a_{k}=0 \forall 0 \neq k \in \mathbb{Z}^{n}$, which yields $\varphi(z)=a_{0}$, a constant.

On the other hand, if $\varphi(z)$ is constant then $\varphi(z)=\varphi\left(z^{2}\right)$, and so we have $A_{\psi} M_{\varphi}=M_{\varphi} A_{\psi}$.

Theorem 6.9. $W A_{\varphi}$ is a slant Toeplitz operator if and only if $\varphi=0$.

Proof. The result is obvious if $\varphi=0$. Conversely suppose $W A_{\varphi}$ is a slant Toeplitz operator with $\varphi(z)=\sum_{\tau \in \mathbb{Z}^{n}} \delta_{\tau} z^{\tau}$. Then by Theorem 4.7, we get $\left\langle W A_{\varphi} e_{k+2 \epsilon_{j}}, e_{t+\epsilon_{j}}\right\rangle=\left\langle W A_{\varphi} e_{k}, e_{t}\right\rangle$ $\forall k, t \in \mathbb{Z}^{n}, 1 \leq j \leq n$.

Now $\left\langle W A_{\varphi} e_{k+2 \epsilon_{j}}, e_{t+\epsilon_{j}}\right\rangle=\left\langle A_{\varphi} e_{k}, e_{2 t+\epsilon_{j}}\right\rangle=\left\langle W M_{\varphi} e_{k}, e_{2 t+\epsilon_{j}}\right\rangle=\left\langle M_{\varphi} e_{k}, e_{4 t+2 \epsilon_{j}}\right\rangle$, and similarly, $\left\langle W A_{\varphi} e_{k}, e_{t}\right\rangle=\left\langle M_{\varphi} e_{k}, e_{4 t}\right\rangle$.

So, $W A_{\varphi}$ is slant Toeplitz implies that $\left\langle M_{\varphi} e_{k}, e_{4 t+2 \epsilon_{i}}\right\rangle=\left\langle M_{\varphi} e_{k}, e_{4 t}\right\rangle$, which yields $\left\langle\varphi(z) z^{k}, z^{4 t+2 \epsilon_{j}}\right\rangle=\left\langle\varphi(z) z^{k}, z^{4 t}\right\rangle$. Consequently $\left\langle\Sigma_{\tau} \delta_{\tau} z^{\tau+k}, z^{4 t+2 \epsilon_{j}}\right\rangle=\left\langle\Sigma_{\tau} \delta_{\tau} z^{\tau+k}, z^{4 t}\right\rangle$ showing that $\delta_{4 t-k+2 \epsilon_{j}}=\delta_{4 t-k} \forall k, t \in \mathbb{Z}^{n}, 1 \leq j \leq n$.

Thus $\delta_{k+2 \epsilon_{j}}=\delta_{k} \forall k \in \mathbb{Z}^{n}, 1 \leq j \leq n$.

So, for each $k \in \mathbb{Z}^{n}$, and $j=1,2, \ldots, n$, we have $\delta_{k}=\delta_{k+2 \epsilon_{j}}=\delta_{k+4 \epsilon_{j}}=\delta_{k+6 \epsilon_{j}}=\cdots$ But $\left|k+2 \lambda \epsilon_{j}\right| \rightarrow \infty$ as $\lambda \rightarrow \infty$, and as $\varphi \in L^{\infty}\left(\mathbb{T}^{n}\right)$ so $\delta_{k+2 \lambda \epsilon_{j}} \rightarrow 0$ as $\lambda \rightarrow \infty$.

Hence $\delta_{k}=0$. As this is true for all $k \in \mathbb{Z}^{n}$, so we must have $\varphi=0$.

Theorem 6.10. For $\varphi, \psi \in L^{\infty}\left(\mathbb{T}^{n}\right)$ the following are equivalent:

(i) $A_{\varphi} A_{\psi}$ is a slant Toeplitz operator. 
AJMS

27,1

90

(ii) $\varphi\left(z^{2}\right) \cdot \psi(z)=0$.

(iii) $A_{\varphi} A_{\psi}=0$.

Proof. (i) $\Leftrightarrow$ (ii) Using Theorem 6.7 we get $A_{\varphi} A_{\psi}=W M_{\varphi} A_{\psi}=W A_{\varphi\left(z^{2}\right) . \psi(z)}$. Therefore by Theorem 6.9, $A_{\varphi} A_{\psi}$ is a slant Toeplitz operator if and only if $\varphi\left(z^{2}\right) \cdot \psi(z)=0$.

(ii) $\Leftrightarrow$ (iii)

As $A_{\varphi} A_{\psi}=W A_{\varphi\left(z^{2}\right) . \psi(z)}$, so $A_{\varphi} A_{\psi}=0$ implies that $W A_{\varphi\left(z^{2}\right) . \psi(z)}$ is a slant Toeplitz operator. Therefore, by Theorem 6.9, $\varphi\left(z^{2}\right) \cdot \psi(z)=0$. Conversely, $\varphi\left(z^{2}\right) \cdot \psi(z)=0$ implies $M_{\varphi\left(z^{2}\right) \cdot \psi(z)}=0$, and so $A_{\varphi} A_{\psi}=W A_{\varphi\left(z^{2}\right) \cdot \psi(z)}=W^{2} M_{\varphi\left(z^{2}\right) \cdot \psi(z)}=0$.

The following theorem gives a necessary and sufficient condition for two slant Toeplitz operators to commute.

Theorem 6.11. For $\varphi, \psi \in L^{\infty}\left(\mathbb{T}^{n}\right), A_{\varphi} A_{\psi}=A_{\psi} A_{\varphi}$ if and only if $\varphi\left(z^{2}\right) \psi(z)=\psi\left(z^{2}\right) \varphi(z)$ for any $z \in \mathbb{Z}^{n}$.

Proof. Suppose $A_{\varphi} A_{\psi}=A_{\psi} A_{\varphi}$. Now $A_{\varphi} A_{\psi}=W M_{\varphi} A_{\psi}$ and by Theorem 6.7, $M_{\varphi} A_{\psi}=$ $A_{\varphi\left(z^{2}\right) \psi(z)}$. Thus, $A_{\varphi} A_{\psi}=A_{\psi} A_{\varphi}$ implies $W A_{\varphi\left(z^{2}\right) \psi(z)}=W A_{\psi\left(z^{2}\right) \varphi(z)}$. In view of Theorem 6.6 we can infer that $W A_{\varphi\left(z^{2}\right) \psi(z)-\psi\left(z^{2}\right) \varphi(z)}=0$, which in turn implies that $W A_{\varphi\left(z^{2}\right) \psi(z)-\psi\left(z^{2}\right) \varphi(z)}$ is a slant Toeplitz operator. Therefore by Theorem 6.9 we get $\varphi\left(z^{2}\right) \psi(z)-\psi\left(z^{2}\right) \varphi(z)=0$ which implies $\varphi\left(z^{2}\right) \psi(z)=\psi\left(z^{2}\right) \varphi(z)$.

Conversely, suppose $\varphi\left(z^{2}\right) \psi(z)=\psi\left(z^{2}\right) \varphi(z)$. Then by Theorem 6.9, we have $W A_{\varphi\left(z^{2}\right) \psi(z)-\psi\left(z^{2}\right) \varphi(z)}$ is slant Toeplitz operator and equals zero. This together with Theorems 6.6 and 6.7 implies that $A_{\varphi} A_{\psi}=A_{\psi} A_{\varphi}$.

Theorem 6.12. $A_{\varphi}^{2}=A_{\varphi}$ if and only if $\varphi=0$. In other words, there are no non-zero idempotents among slant Toeplitz operators.

Proof. Suppose $A_{\varphi}^{2}=A_{\varphi}$. Then $A_{\varphi}^{2}=A_{\varphi} \cdot A_{\varphi}$ is slant Toeplitz, so that by Theorem 6.10 we have $A_{\varphi}^{2}=0$. Thus $A_{\varphi}=0=W A_{\varphi}$. This in turn implies that $W A_{\varphi}$ is slant Toeplitz, and by Theorem 6.9 we get $\varphi=0$.

Conversely, $\varphi=0 \Rightarrow M_{\varphi}=0 \Rightarrow A_{\varphi}=W M_{\varphi}=0 \Rightarrow A_{\varphi}^{2}=A_{\varphi}$.

Theorem 6.13. $A_{\varphi}^{*}$ is a slant Toeplitz operator if and only if $\varphi=0$.

Proof. If $\varphi=0$ then $A_{\varphi}=0=A_{\varphi}^{*}$ and so $A_{\varphi}^{*}$ is slant Toeplitz.

Conversely, suppose $A_{\varphi}^{*}$ is slant Toeplitz. Then, by Theorem 4.7, $\left\langle A_{\varphi}^{*} e_{k+2 \epsilon_{j}}, e_{t+\epsilon_{j}}\right\rangle=\left\langle A_{\varphi}^{*} e_{k}, e_{t}\right\rangle \forall k, t \in \mathbb{Z}^{n}, 1 \leq j \leq n$. This implies that

$\left\langle A_{\varphi} e_{t+\epsilon_{j}}, e_{k+2 \epsilon_{j}}\right\rangle=\left\langle A_{\varphi} e_{t}, e_{k}\right\rangle \forall k, t \in \mathbb{Z}^{n}, 1 \leq j \leq n$, which in turn implies $\left\langle W M_{\varphi} e_{t+\epsilon_{j}}\right.$, $\left.e_{k+2 \epsilon_{j}}\right\rangle=\left\langle W M_{\varphi} e_{t}, e_{k}\right\rangle \forall k, t \in \mathbb{Z}^{n}, \quad 1 \leq j \leq n$.

Taking $\varphi(z)=\sum_{\tau \in \mathbb{Z}^{n}} a_{\tau} z^{\tau}$ in the above expression, we get

$\left\langle\sum_{\tau} a_{\tau} z^{\tau+t+\epsilon_{j}}, z^{2 k+4 \epsilon_{j}}\right\rangle=\left\langle\sum_{\tau} a_{\tau} z^{\tau+t}, z^{2 k}\right\rangle \quad \forall k, t \in \mathbb{Z}^{n}, 1 \leq j \leq n$, which yields $a_{2 k-t+3 \epsilon_{j}}=a_{2 k-t} \forall k$, $t \in \mathbb{Z}^{n}, 1 \leq j \leq n$

Thus, $a_{k+3 \epsilon_{j}}=a_{k} \forall k \in \mathbb{Z}^{n}, 1 \leq j \leq n$, and as in Theorem 6.9, this implies that $\varphi=0$.

Corollary 6.14. There is no non-zero self adjoint slant Toeplitz operator.

Theorem 6.15. $A_{\varphi}$ is hyponormal if and only if $\varphi=0$.

Proof. $A_{\varphi}$ is hyponormal if and only if $\left\|A_{\varphi} f\right\|^{2} \geq\left\|A_{\varphi}^{*} f\right\|^{2} \forall f \in L^{2}\left(\mathbb{T}^{n}\right)$. Equivalently, $\|W(\varphi(z) f(z))\|^{2} \geq\left\|\overline{\varphi(z)} W^{*} f(z)\right\|^{2} \forall f \in L^{2}\left(\mathbb{T}^{n}\right)$

Let $\varphi(z)=\sum_{k \in Z^{n}} a_{k} z^{k}$. If $\varphi=0$ then clearly $A_{\varphi}=0$ is hyponormal. For the converse we consider the following two cases. 
Case 1: Let $f(z)=1=e_{0}(z)$. Then by Remark 4.4(ii), we have $W^{*} f(z)=1$

$$
\begin{aligned}
\text { So } A_{\varphi} \text { is hyponormal } & \Rightarrow\left\|W\left(\sum_{k \in \mathbb{Z}^{n}} a_{k} z^{k}\right)\right\|^{2} \geq\left\|\sum_{k \in \mathbb{Z}^{n}} \bar{a}_{k} z^{-k}\right\|^{2} \\
& \Rightarrow\left\|\sum_{k \in \mathbb{Z}^{n}} a_{2 k} z^{k}\right\|^{2} \geq \sum_{k \in Z^{n}}\left|\bar{a}_{k}\right|^{2} \\
& \Rightarrow \sum_{k \in \mathbb{Z}^{n}}\left|a_{2 k}\right|^{2} \geq \sum_{k \in \mathbb{Z}^{n}}\left|a_{k}\right|^{2} \\
& \Rightarrow \sum_{k \in \mathbb{Z}^{n}, k o d d}\left|a_{k}\right|^{2} \leq 0 \\
& \Rightarrow a_{k}=0 \forall k \in \mathbb{Z}^{n}, k \text { odd }
\end{aligned}
$$

Therefore, $\varphi(z)=\sum_{k \in Z^{n}} a_{2 k} z^{2 k}$.

Case 2: Let $f(z)=z$ so that $W^{*} f(z)=z^{2}$, and

$$
\begin{aligned}
A_{\varphi} \text { is hyponormal } & \Rightarrow\left\|W\left(\sum_{k \in \mathbb{Z}^{n}} a_{2 k} z^{2 k+1}\right)\right\|^{2} \geq\left\|\sum_{k \in \mathbb{Z}^{n}} \bar{a}_{2 k} z^{2-2 k}\right\|^{2} \\
& \Rightarrow 0 \geq \sum_{k \in \mathbb{Z}^{n}}\left|a_{2 k}\right|^{2}, \text { by Remark 4.4(i) } \\
& \Rightarrow a_{2 k}=0 \forall k \in \mathbb{Z}^{n} .
\end{aligned}
$$

Thus, $\varphi=0$.

Theorem 6.16. A slant Toeplitz operator cannot be an isometry.

Proof. Let, if possible $A_{\varphi}$ be an isometry. Then $A_{\varphi}^{*} A_{\varphi}=I$. Or equivalently, $\left\|A_{\varphi} f\right\|=\|f\| \forall f \in L^{2}\left(\mathbb{T}^{n}\right)$

By Theorem 6.3, $A_{\varphi} A_{\varphi}^{*}=M_{\psi}$ where $\psi=W|\varphi|^{2}$

Also by Corollary 6.4, $\|\psi\|_{\infty}=\left\|A_{\varphi}\right\|^{2}=\left\|A_{\varphi}^{*} A_{\varphi}\right\|=1$. Thus, $\left\|W|\varphi|^{2}\right\|_{\infty}=1$

Let $\varphi(z)=\sum_{k \in Z^{n}} a_{k} z^{k}$. Then $\|\varphi\|=\left(\sum_{k \in \mathbb{Z}^{n}}\left|a_{k}\right|^{2}\right)^{\frac{1}{2}}=\left[\sum_{t \in S}\left(\sum_{k \in Z^{n}}\left|a_{2 k+t}\right|^{2}\right)\right]^{\frac{1}{2}}$, by Lemma 5.2. Therefore,

$$
\left\|W|\varphi|^{2}\right\|_{\infty}=\left\|W M_{\varphi} \bar{\varphi}\right\|=\left\|A_{\varphi} \bar{\varphi}\right\|=\|\bar{\varphi}\|=\|\varphi\|=\left[\sum_{t \in S}\left(\sum_{k \in Z^{n}}\left|a_{2 k+t}\right|^{2}\right)\right]^{\frac{1}{2}}
$$

For $t \in S, A_{\varphi} \bar{z}^{t}=W M_{\varphi} \bar{z}^{t}=W \sum_{k \in \mathbb{Z}^{n}} a_{k} z^{k-t}=W \sum_{\tau \in \mathbb{Z}^{n}} a_{\tau+t} z^{\tau}$. So by Remark 4.4(i), $A_{\varphi} \bar{z}^{t}=$ $\sum_{\tau \in Z^{n}} a_{2 \tau+t} z^{\tau}$. Combining this along with the fact that $\left\|A_{\varphi} \bar{z}^{t}\right\|^{2}=\left\|\bar{z}^{t}\right\|^{2}=1$, we get $\| \sum_{\tau \in Z^{n}}$ $a_{2 \tau+t} z^{\tau} \|^{2}=1$, which implies that $\sum_{\tau \in Z^{n}}\left|a_{2 \tau+t}\right|^{2}=1$.

Using this in Eq. (6.2) we get $\left\|W|\varphi|^{2}\right\|_{\infty}=2^{\frac{h}{2}}$, since $o(S)=2^{n}$.

So, $\left\|W|\varphi|^{2}\right\|_{\infty}=1$ means $2^{\frac{n}{2}}=1$, which is only possible if $n=0 . n$ being a positive integer this is not possible, and so we conclude that $A_{\varphi}$ cannot be an isometry.

Theorem 6.17. $A_{\varphi}$ is compact if and only if $\varphi=0$. 
AJMS

27,1

92
Proof. We know that the set of compact operators on a Hilbert space $H$ is an ideal of the space of bounded linear operators on $H$. Thus,

$A_{\varphi}$ is compact $\Rightarrow A_{\varphi} M_{z^{t}}$ is compact $\Rightarrow\left(A_{\varphi} M_{z^{t}}\right)^{*}$ is compact $\forall t \in S$.

For $t \in S,\left(A_{\varphi} M_{z^{t}}\right)^{*}=\left(W M_{\varphi} M_{z^{t}}\right)^{*}=\left(W M_{z^{t} \varphi}\right)^{*}=A_{z^{t} \varphi^{*}}^{*}$

So, $W\left(A_{\varphi} M_{z^{t}}\right)^{*}=W A_{z^{t} \varphi}^{*}=M_{\psi}$, where $\psi=W\left(\overline{z^{t} \varphi}\right)$ by Theorem 5.9

But $A_{\varphi} M_{z^{t}}$ is compact implies that $W\left(A_{\varphi} M_{z^{t}}\right)^{*}=M_{\psi}$ is compact, and consequently $\psi=0$.

Hence $\left\langle W\left(\overline{z^{t} \varphi}\right), z^{m}\right\rangle=\left\langle\psi, z^{m}\right\rangle=0 \forall m \in \mathbb{Z}^{n}$, and so $\left\langle\bar{\varphi}, z^{2 m+t}\right\rangle=0 \forall m \in \mathbb{Z}^{n}, t \in S$. Thus, if $\varphi(z)=\sum_{k \in \mathbb{Z}^{n}} a_{k} z^{k}$, then we must have $a_{2 m+t}=0 \forall m \in \mathbb{Z}^{n}, t \in S$, and now, by Lemma 5.2, we get $a_{k}=0 \forall k \in \mathbb{Z}^{n}$, so that $\varphi=0$.

\section{Remarks}

In Section 3 we have shown that an operator on $H^{2}\left(\mathbb{D}^{n}\right)$ is a Toeplitz operator if and only if it can be represented as a Toeplitz matrix of level $n$. Using this relation, we can define the operator $B_{\varphi}$ on $H^{2}\left(\mathbb{D}^{n}\right)$ as $B_{\varphi}=W T_{\varphi}$, calling it the compression of the slant Toeplitz operator to the Hardy space of the polydisk. A study of the properties of $B_{\varphi}$ vis-a-vis that of $A_{\varphi}$ should yield interesting results. For $H^{2}(\mathbb{D})$, similar studies have been conducted in [2], Section 3 [18], and [22].

\section{References}

[1] S.C. Arora, R. Batra, On generalized slant Toeplitz operators, Indian J. Math. 45 (2) (2003) 121-134.

[2] S.C. Arora, R. Batra, Generalised slant Toeplitz operators on $H^{2}$, Math. Nachr. 278 (4) (2005) 347-355.

[3] S.C. Arora, J. Bhola, Essentially slant Toeplitz operators, Banach J. Math. Anal. 3 (2) (2009) 1-8.

[4] B. Choe, H. Koo, Y. Lee, Commuting Toeplitz operators on the polydisk, Trans. Amer. Math. Soc. 356 (2004) 1727-1749.

[5] G. Datt, R. Aggarwal, On a generalization of slant Toeplitz operators, Gen. Math. 21 (2) (2013) 57-69.

[6] X. Ding, Products of Toeplitz operators on the polydisk, Integral Equations Operator Theory 45 (2003) 389-403.

[7] X. Ding, S. Sun, D. Zheng, Commuting Toeplitz opertors on the bidisk, J. Funct. Anal. 263 (2012) 3333-3357.

[8] C. Gu, Some algebraic properties of Toeplitz and Hankel operators on polydisk, Arch. Math. (Basel) 80 (2003) 393-405.

[9] C. Gu, J. Hendericks, D. Rutherford, Hyponormality of block Toeplitz operators, Pacific J. Math. 223 (1) (2006) 95-111.

[10] M. Hazarika, S. Marik, Hyponormality of generalised slant weighted Toeplitz operators with polynomial symbols, Arab. J. Math. 7 (2018) 9-19.

[11] M.C. Ho, Properties of slant Toeplitz operators, Indiana Univ. Math. J. 45 (1996) 843-862.

[12] M.C. Ho, Adjoints of slant Toeplitz operators, Integral Equations Operator Theory 29 (3) (1997) 301-312.

[13] M.C. Ho, Adjoints of slant Toeplitz operators RN2, Integral Equations Operator Theory 41 (2) (2001) 179-188.

[14] L. Kong, Y. Lu, Commuting Toeplitz operators on the Hardy space of the polydisk, Acta Math. Sin. (Engl. Ser.) 31 (2) (2015) 695-702. 
[15] S. Koyuncu, K.R. Bowman, Product of several two-level Toeplitz operators, JMR 5 (2) (2013) 61-64.

[16] Y. Lee, Commuting Toeplitz operators on the Hardy space of the polydisk, Proc. Amer. Math. Soc. 138 (2010) 189-197.

[17] C. Liu, Y. Lu, Product and commutativity of slant Toeplitz operators, J. Math. Res. Appl. 33 (1) (2013) 122-126.

[18] Y. Lu, C. Liu, J. Yang, Commutativity of kth order slant Toeplitz operators, Math. Nachr. 283 (9) (2010) 1304-1313.

[19] Y. Lu, Y. Shi, Hyponormal Toeplitz operators on the polydisk, Acta Math. Sin. (Engl. Ser.) 28 (2) (2012) 333-348.

[20] W. Rudin, Function Theory in Polydiscs, W. A. Benjamin Inc., New York-Amsterdam, 1969.

[21] S. Sun, D. Zheng, Toeplitz operators on the polydisk, Proc. Amer. Math. Soc. 124 (1996) 3351-3356.

[22] T. Zegeye, S.C. Arora, The compression of a slant Toeplitz operator to $H^{2}$, Indian J. Pure Appl. Math. 32 (2) (2001) 221-226.

[23] T. Zegeye, S.C. Arora, M.P. Singh, On slant Toeplitz operators, Indian J. Math. 42 (3) (2000) 379-385.

\section{Corresponding author}

Munmun Hazarika can be contacted at: munmun@tezu.ernet.in
Toeplitz and slant Toeplitz operators 